\title{
Bee Propolis, Bee Bread, and Royal Jelly: Proximate Analysis, Fatty Acid Composition, Nutritional Quality, and Anti-Amylase Activity
}

Masoomeh Fallah

Shiraz University

\section{Fatemeh Najafi}

Shiraz University

Gholamreza Kavoosi ( $\square$ ghkavoosi@shirazu.ac.ir)

Shiraz University

\section{Research Article}

Keywords: Bee bread, Bee propolis, Royal jelly, Fatty acids, Nutritional quality

Posted Date: September 29th, 2021

DOI: https://doi.org/10.21203/rs.3.rs-927023/v1

License: (c) (i) This work is licensed under a Creative Commons Attribution 4.0 International License. Read Full License 


\section{Abstract}

This work explores the proximate composition, fatty acid profile, nutritional quality, and anti-amylase activity of propolis, royal jelly, and bee bread. The differential FTIR patterns of propolis, royal jelly, and bee bread reflect these products have different proximate compositions and nutritional properties. The values for carbohydrate, fat, and protein values of be products are similar to egg and soya. The primary fatty acids bee bread and bee propolis are palmitic, linolenic, oleic, linoleic, myristic, and docosanoic acids. The primary fatty acids in royal jelly are 2-dodecenedioic, 10-hydroxy-2-decenoic, decanedioic, linoleic, 10hydroxydecanoic acid 3-hydroxy-decanoic acids, respectively. The propolis, bee bread, and royal jelly have well-balanced saturated, unsaturated, monounsaturated, polyunsaturated, omega-3, and omega- 6 fatty acids. Their nutritional quality, including omega-6/omega-3, thrombogenicity, atherogenicity, hypocholesterolemic, nutritional value, and peroxidizability indexes, are similar to egg and soya. Fatty acids inhibit amylase by increasing $\mathrm{Km} / \mathrm{Vmax}$ and decreasing Vmax and $\mathrm{Km}$ through an un-competition or noncompetition strategy. Molecular docking, ultraviolet absorption, and fluorescence quenching analysis reveal that fatty acids interact with amino acid residues of amylase through Van der Waals and hydrogen bonds interactions. Functional fatty acids from bee products can be used in a number of food supplements, food ingredients, and medications to provide carbohydrate-degrading enzymes.

\section{Introduction}

The increasing shortage of world food and pharmaceutical sources and the growing world population compels us to search for alternative resources such as bee products. Bee propolis, royal jelly, pollen, and bee bread possess nutritional value and health-protecting properties and recommend a superfood for human food and feed. Propolis, bee bread, and royal jelly are applied in the management of different illnesses since they have several biological properties including, antioxidant, anti-fungal, anti-bacterial, antiseptic, anti-cancer, and anti-inflammatory, depending on chemical composition and region of collection. Current research demonstrated the advantageous properties of propolis in the management of obesity, diabetes mellitus, and dyslipidemia ${ }^{1}$. Bee propolis is a sticky mixture of wax, resin, protein, fatty acid, polysaccharides, and exudate from the plant's flower produced by mixing with the bee salvia. Commonly, propolis contains resins (50-60\%), waxes (30-40\%), essential oil (5.0-10\%), and other constituents, including proteins, amino acids, free fatty acids, vitamins, and micronutrients, depending on plant sources ${ }^{2}$. Bee bread is a mixture of plant flower pollen, wax, honey, and salivary gland secretion of bees compacted into honeycombs. Bee bread mixture is a perfectly complete food, has high energy, and is a nutrient resource for bee larvae and adult bees ${ }^{3}$. The components of bee bread encompass proteins and amino acid (8.0-28\%), carbohydrates (15-45\%), lipids and fatty acids (7.0-15\%), vitamins, minerals, and antioxidant compounds such as flavonoids, and phenolic acids, depending on the plant's sources and collationed region 4 .

Royal jelly is a yellowish and creamy emulsion of protein (8.0-22\%), amino acid (1.5-3.5\%), sugars (7.0$52 \%$, primarily consists of fructose and glucose), lipids ( $7-33 \%$, short hydroxyl fatty acids, and dicarboxylic acids), and polyphenols, flavonoids, and vitamins ${ }^{5}$. This high-energy emulsion is a valuable food for the 
queen in adult and larvae stages and young bee larvae. One of the main characteristics of royal jelly emulsion is hydroxylated fatty acids and dicarboxylic acids. The primary hydroxylated fatty acids in royal jelly are 10-hydroxydecanoic and 10-hydroxy-2-decanoic acids with various biological activities ${ }^{6}$. Proximate and nutrient analysis of bee products plays a crucial role in assessing their nutritional significance. As various bee products are also used as food and medicinal ingredients, evaluating their nutritional effectiveness can help understand the worth of these products. As far as herbal drug standardization is concerned, WHO also emphasizes the need and importance of determining proximate and macronutrients analysis. Soybean ${ }^{7}$ and egg $^{8}$ are significant sources of protein and fatty acid for the human diet, recommended by WHO/FDA.

The fundamental goal of current work is to determine the nutritional properties of bee bread, bee propolis, and royal jelly through examining their proximate composition and comparing them with egg (as an animal resource) and soybean (as a plant resource). The lipid nutritional quality and fatty acid composition of bee bread, bee propolis, and royal jelly were determined and compared with egg and soybean fatty acids. The data matrix of the fatty acid composition and nutritional value in the samples were subjected to principal component analysis to illustrate the apparent discrepancy of distribution of these components among the samples. The researcher examines the anti-amylase activity of fatty acids from bee bread, bee propolis, and royal jelly. Double reciprocal Lineweaver-Burk plots will investigate the mechanism of inhibition of fatty acid on the amylase activity. The interactions between amylase and fatty acids were analyzed using ultraviolet absorption spectroscopy and fluorescence quenching spectroscopy. Furthermore, molecular modeling does use to determine the interaction between fatty acids and amylase.

\section{Materials And Methods}

\subsection{Proximate composition of bee product}

Bee products including bee propolis, bee bread, and royal jelly are prepared from beekeepers in Marvdasht region of Fars province (Fars, Iran). The predominant vegetation in this area is wild almond. To evaluate the differential chemical components of bee propolis, bee bread, and royal jelly, Fourier transforms infrared spectroscopy (FT-IR), in the range between $4000-400 \mathrm{~cm}^{-1}$, performed utilizing Bruker FTIR spectrophotometer (Germany). The moisture content of bee products determines by drying the samples at $105^{\circ} \mathrm{C}$ for two $\mathrm{h}$ through a vacuum oven (model: BM55E, Fan Azma Gostar, Iran). The bee samples were heated at $550^{\circ} \mathrm{C}$ for five $\mathrm{h}$ in electric ovens (model: FM2P, Fan Azma Gostar, Iran) for ash determination. The phenol-sulfuric acid method applies to assessing total carbohydrate content ${ }^{9}$. According to the Bradford method, total protein content in the bee products evaluates through Coomassie brilliant blue $\mathrm{G}-250^{10}$. The sulfuric acid-phosphoric acid-vanillin reagent uses to quantify total lipid and fatty acids ${ }^{11}$. The following formula measured the total energy content of the bee product: Total energy $=(4.0 \mathrm{kcal} / \mathrm{g} \times \mathrm{g}$ protein $)+(9.0$ $\mathrm{kcal} / \mathrm{g} \times \mathrm{g}$ fat $)+(4.0 \mathrm{kcal} / \mathrm{g} \times \mathrm{g}$ carbohydrate $)$.

Bee products $(1.0 \mathrm{~g})$ mix with pepsin $(2 \mathrm{mg} / 100 \mathrm{ml})$ in $7.5 \mathrm{mM} \mathrm{HCl}(100 \mathrm{ml})$. These mixtures incubate at $40^{\circ} \mathrm{C}$ for $15 \mathrm{~h}$ with continuous shaking. The undigested protein content in the samples before and after 
pepsin treatment was determined as mentioned above. The ratio of digested protein to the original protein reflects the digestibility level. The protein digestibility level expresses as the digested protein $(\mathrm{g})$ per $100 \mathrm{~g}$ of total protein in the raw materials.

\subsection{Fatty acid preparation}

The bee products biomass wash digested for 48 hours using hydrochloric acid: normal saline: methanol (2:1:1) solution at $60^{\circ} \mathrm{C}$. Hexane was applied to the digestion solution and vortexed for 10 minutes to separate fatty acids. The fatty acids in the hexane phase separate from the bottom phase. The fatty acid chemical composition was examined by gas chromatography/mass spectrometry (GC-MS). A rotary evaporator uses to evaporate the hexane phase at ambient temperature. The residual fatty acids dissolve in ethanol for further experiments ${ }^{12}$.

\subsection{Fatty acid profiling by GC-MS}

Fatty acid profile attains by means the gas chromatograph (Agilent 7890B GC 7955A MSD) equipped with a single quadrupole mass spectrometer. The standard column for fatty acid determination was the silica capillary HP-5MS column $(30 \mathrm{~m} \times 0.25 \mathrm{~mm}, 0.25 \mu \mathrm{m})$. Helium at a flow rate of $1.0 \mathrm{ml} / \mathrm{min}$ applies as carrier gas. Interface and ion source temperatures were $300^{\circ} \mathrm{C}$ and $250^{\circ} \mathrm{C}$, respectively. The oven temperature program was $80-250^{\circ} \mathrm{C}$ as follow: $80^{\circ} \mathrm{C}$ for four min, rise at $20^{\circ} \mathrm{C} / \mathrm{min}$ to $140^{\circ} \mathrm{C}$, grow at ten ${ }^{\circ} \mathrm{C} / \mathrm{min}$ to $250^{\circ} \mathrm{C}$, then hold at $250^{\circ} \mathrm{C}$ for $10 \mathrm{~min}$. The types of fatty acids are determined by comparison the fragmentation patterns of the associated peaks with these suggested in the libraries of Wily and $\mathrm{NIST}^{13}$.

\subsection{Indices of lipid nutritional quality}

The nutritional quality of the bee products measures by calculating the total saturated fatty acids (SFA), total unsaturated fatty acids (UFA), total monounsaturated fatty acids (MUFA), total polyunsaturated fatty acids (PUFA), total omega-3, and total omega- $6^{14}$. Based on total SFA, total UFA, total MUFA, and total PUFA, we calculate hypocholesterolemic / hypercholesterolemic index $(\mathrm{H} / \mathrm{H})$, thrombogenicity index $(\mathrm{TI})$, atherogenicity index (Al), PUFAs/SFAs (P/S), omega-6 PUFAs/omega-3 PUFAs index, nutritive value index $(\mathrm{NVI})$, and peroxidizability index $(\mathrm{PI})^{15}$.

\subsection{Amylase activity inhibition}

To the porcine pancreatic a-amylase (EC: 3.2.1.1, $5.0 \mathrm{U} / \mathrm{mg}$ ) solution (1.0 Unit/ml), different concentrations $(0.3 .0 .6,0.9 \mathrm{mg} / \mathrm{ml})$ of fatty acid and acarbose solutions was added, then incubated at $37^{\circ} \mathrm{C}$ for 20 minutes. To the amylase-inhibitor, starch solution $(5.0 \mathrm{mg} / \mathrm{ml}$ in $100 \mathrm{mM}$ phosphate buffer, $\mathrm{pH} 7)$ add. The amylase-inhibitor-starch mixture incubates for $20 \mathrm{~min}$ at $37^{\circ} \mathrm{C}$. By adding $0.02 \mathrm{ml}$ of $1.0 \mathrm{M} \mathrm{HCl}$ to the mixture, the amylase reaction stops. To assess undigested starch, iodine solution pours on the amylase reaction. The amylase reaction dilutes with distilled water, and the absorbance of samples is recorded at $580 \mathrm{~nm}$. The activity (velocity) of amylase in the absence and presence of acarbose or fatty acid was calculated via monitoring decomposition of starch per min by measuring light absorbance at $580 \mathrm{~nm}$ for 30 min at an interval of $3.0 \mathrm{~min}$. The slope of starch decomposition versus time plot represented the amylase activity and expressed as $\mu \mathrm{g} / \mathrm{min}$. The kinetic parameters of amylase activity were obtained from the Lineweaver-Burk curve that plotted based on $1 / \mathrm{V}$ versus $1 /[$ starch] in the absence and presence of fatty acid 
or acarbose (0.3. 0.6, $0.9 \mathrm{mg} / \mathrm{ml})$. The kinetic parameters like $\mathrm{Km} / \mathrm{Vmax}, \mathrm{Km}$, and Vmax and the type of inhibition attained from the Lineweaver-Burk plot ${ }^{12}$.

\subsection{Fluorescence and ultraviolet spectroscopy analysis}

The interactions between amylase and fatty acid investigated using ultraviolet absorption spectroscopy (UV1280, Shimadzu, Japan) and fluorescence quenching spectroscopy (Varian Cary Eclipse, Agilent, USA $)^{16}$. Amylase solution $(5.0 \mathrm{ml}$ of $500 \mu \mathrm{g} / \mathrm{ml})$ was prepared in phosphate buffer $(100 \mathrm{mM}, \mathrm{pH}=7)$. To the enzymatic solutions, $5.0 \mathrm{ml}$ of different concentrations $(0.3 .0 .6,0.9 \mathrm{mg} / \mathrm{ml})$ of acarbose or fatty acid were added. The reaction mixtures incubate at ambient temperature for $10 \mathrm{~min}$. The UV absorption spectrum of the amylase-inhibitor mixture was recorded from 200 to $400 \mathrm{~nm}$ using quartz cuvettes at room temperature. The intrinsic fluorescence absorption spectrum of the amylase-inhibitor solutions records at $280 \mathrm{~nm}$ for excitation wavelength and $290-500 \mathrm{~nm}$ for emission wavelengths.

\subsection{Molecular dicking}

Three-dimensional structure of the amylase downloads from the protein data bank (5U3A, PDB). Threedimensional structures of fatty acid and acarbose download from the chemspider website. AutoDock vina platform was used to examine interactions between amylase and ligands. The evaluation of the docked positions and amylase-ligand interactions and docking scores (binding free energy) was attained employing PYMOL software ${ }^{12}$.

\subsection{Statistical analysis}

All results show mean values \pm standard deviations. The data were analyzed with a one-way analysis of variance (ANOVA) using SPSS software (SPSS Inc., Chicago, IL, USA). The significant differences between samples were examined by the Tukey test at $P<0.05$. Minitab software was used to perform the principal component analysis (PCA).

\section{Results And Discussions}

\subsection{Proximate composition}

The differential FTIR patterns from bee bread, royal jelly, and bee propolis reflect the different components in these products (Fig. 1). The bands in the $3700-3100 \mathrm{~cm}^{-1}$ might connect to stretching vibration of $\mathrm{OH}$ groups of water or aromatic compounds. The peaks in the $2900-2700 \mathrm{~cm}^{-1}$ may link to the stretching vibration of $\mathrm{CH}, \mathrm{CH} 2$, and $\mathrm{CH} 3$ groups of lipid and fatty acids. The bands in $1300-900 \mathrm{~cm}^{-1}$ related to the stretching of $\mathrm{C}=\mathrm{O}$ of amides, $\mathrm{C}=\mathrm{C}$ of aromatic, $\mathrm{N}-\mathrm{H}$ of amines, or carboxyl groups in proteins. The bands at $1100-500 \mathrm{~cm}^{-1}$ are due to vibration of polysaccharides, including symmetric stretching of $\mathrm{C}-\mathrm{O}-\mathrm{C}$ and $\mathrm{OH}$ groups $^{17}$.

The chemical composition (moisture content, ash, carbohydrate, fat, and protein ad energy values) of bee bread, bee propolis, and royal jelly product were compared with egg and soya and summarized in Table 1. Principal component analysis showed that the sum of the first and second major components accounted 
for $94.4 \%$ total variance of the changes, with the first major component (PC1) accounting for $65.7 \%$ (eigenvalue $=4.60$ ) and the second major component $(P C 2)$ for $28.7 \%$ (eigenvalue $=2.00)$ (Fig. 2). The first principal component (PC1) is positively correlated with energy (0.458), ash (0.444), fatty acid (0.351), and protein (0.318). Therefore, increasing energy, ash, fatty acid, and protein values increase the value of the first principal component. PC1 is negatively correlated with digestibility $(-0.421)$ and moisture $(-0.414)$. The second principal component (PC2) is positively correlated with sugar $(0.670)$. Therefore, increasing the values of sugar increase the value of the PC2. PC2 is negatively correlated with digestibility $(-0.215)$, moisture $(-0.279)$, protein $(-0.462)$ and fatty acid $(-0.456)$. Royal jelly in the first quarter is not closely related to the measured parameters. The bee bread and propolis in the second quarter are the most related to sugar and energy. Egg in the 3rd quarter is most associated with digestibility and moisture. In the 4th quarter, soya is most associated with a fatty acid, protein, and ash ${ }^{18}$.

Table 1

Proximate analysis of bee propolis, bee bread and royal jelly in comparison with egg and soya.

\begin{tabular}{|c|c|c|c|c|c|}
\hline Chemicals & $\begin{array}{l}\text { Bee } \\
\text { propolis }\end{array}$ & Bee bread & Royal jelly & Egg & Soya \\
\hline Moisture content $(\mathrm{g} / 100 \mathrm{~g})$ & $\begin{array}{l}15.45 \pm \\
1.14 \mathrm{~b}\end{array}$ & $\begin{array}{l}11.81 \pm \\
0.90 \mathrm{a}\end{array}$ & $\begin{array}{l}25.50 \pm \\
1.90 \mathrm{c}\end{array}$ & $\begin{array}{l}72.00 \pm \\
3.0 \mathrm{~b}\end{array}$ & $\begin{array}{l}9.80 \pm \\
0.75 a\end{array}$ \\
\hline Ash $(\mathrm{g} / 100 \mathrm{~g})$ & $\begin{array}{l}2.76 \pm \\
0.22 \mathrm{~b}\end{array}$ & $\begin{array}{l}2.11 \pm \\
0.15 b\end{array}$ & $\begin{array}{l}1.46 \pm \\
0.12 \mathrm{a}\end{array}$ & $\begin{array}{l}1.10 \pm \\
0.05 a\end{array}$ & $\begin{array}{l}3.37 \pm \\
0.27 c\end{array}$ \\
\hline Protein $(\mathrm{g} / 100 \mathrm{~g})$ & $\begin{array}{l}11.00 \pm \\
0.85 a\end{array}$ & $\begin{array}{l}18.50 \pm \\
1.40 \mathrm{~b}\end{array}$ & $\begin{array}{l}21.70 \pm \\
1.55 \mathrm{~b}\end{array}$ & $\begin{array}{l}13.70 \pm \\
1.11 \mathrm{a}\end{array}$ & $\begin{array}{l}39.75 \pm \\
1.50 c\end{array}$ \\
\hline Fatty acid $(\mathrm{g} / 100 \mathrm{~g})$ & $\begin{array}{l}13.34 \pm \\
1.16 \mathrm{a}\end{array}$ & $\begin{array}{l}11.40 \pm \\
0.84 a\end{array}$ & $\begin{array}{l}12.50 \pm \\
1.00 \mathrm{a}\end{array}$ & $\begin{array}{l}11.50 \pm \\
0.87 \mathrm{a}\end{array}$ & $\begin{array}{l}33.86 \pm \\
2.22 \mathrm{~b}\end{array}$ \\
\hline Sugar $(g / 100 g)$ & $\begin{array}{l}57.15 \pm \\
3.25 \mathrm{~d}\end{array}$ & $\begin{array}{l}55.60 \pm \\
3.30 \mathrm{~d}\end{array}$ & $\begin{array}{l}38.35 \pm \\
2.45 c\end{array}$ & $\begin{array}{l}1.40 \pm \\
0.20 \mathrm{a}\end{array}$ & $\begin{array}{l}12.66 \pm \\
1.12 b\end{array}$ \\
\hline Energy (kcal/100g) & $\begin{array}{l}392.66 \pm \\
14 \mathrm{c}\end{array}$ & $\begin{array}{l}399.00 \pm \\
12 \mathrm{c}\end{array}$ & $\begin{array}{l}352.70 \pm \\
11 \mathrm{~b}\end{array}$ & $\begin{array}{l}163.90 \pm \\
6.7 a\end{array}$ & $\begin{array}{l}505.38 \pm \\
15 d\end{array}$ \\
\hline $\begin{array}{l}\text { Digestibility (g/100g raw } \\
\text { material) }\end{array}$ & $\begin{array}{l}5.69 \pm \\
0.45 a\end{array}$ & $\begin{array}{l}11.56 \pm \\
0.85 \mathrm{~b}\end{array}$ & $\begin{array}{l}15.85 \pm \\
1.20 c\end{array}$ & $\begin{array}{l}11.25 \pm \\
0.75 \mathrm{~b}\end{array}$ & $\begin{array}{l}21.35 \pm \\
1.55 \mathrm{~d}\end{array}$ \\
\hline Digestibility (g/100g protein) & $\begin{array}{l}51.68 \pm \\
2.84 a\end{array}$ & $\begin{array}{l}62.49 \pm \\
3.70 \mathrm{~b}\end{array}$ & $\begin{array}{l}73.04 \pm \\
4.30 \mathrm{c}\end{array}$ & $\begin{array}{l}82.12 \pm \\
4.76 \mathrm{~d}\end{array}$ & $\begin{array}{l}53.71 \pm \\
3.60 \mathrm{a}\end{array}$ \\
\hline
\end{tabular}

\subsection{Fatty acid composition}

The fatty acid composition of bee bread, bee propolis, and royal jelly product were stated in Table 2 . Linoleic, palmitic, oleic, a-linolenic, behenic, and myristic acids are the primary fatty acids in propolis. Linolenic, palmitic, a-linolenic, myristic oleic, and behenic acids are the primary fatty acids in bee bread. alinolenic, 2-dodecenedioic, 10-hydroxy-2-decenoic, decanedioic, linoleic, 10-hydroxydecanoic, 3-hydroxydecanoic, palmitic, 11-hydroxy-dodecanoic, palmitoleic, and oleic acids are primary fatty acids in the royal 
jelly (Table 2). The fatty acid composition of fatty acids from bee bread, bee propolis, royal jelly from other geographical regions differs. Fatty acids such as; capric, linoleic, palmitic, stearic, linolenic, oleic, behenic, arachidic, lauric, decanoic, dodecanoic, tetradecanoic, octadecenoic, tetracosanoic, eicosanoic, and hexacosanoic acids, are reported in bee products ${ }^{19}$. 
Table 2

Fatty acid composition (percent of area) of fatty acid from bee bread, bee propolis, and royal jelly in comparison with egg and soya.

\begin{tabular}{|c|c|c|c|c|c|c|c|}
\hline Common Name & Systematic Name & $\begin{array}{l}\text { Lipid } \\
\text { Numbers }\end{array}$ & $\begin{array}{l}\text { Bee } \\
\text { propolis }\end{array}$ & $\begin{array}{l}\text { Bee } \\
\text { bread }\end{array}$ & $\begin{array}{l}\text { Royal } \\
\text { jelly }\end{array}$ & Egg & Soya \\
\hline Levulinic acid & Levulinic acid & $\mathrm{C} 5 \mathrm{H} 803$ & $\begin{array}{l}0.00 \pm \\
0.00 \mathrm{a}\end{array}$ & $\begin{array}{l}0.75 \\
\pm \\
0.03 a\end{array}$ & $\begin{array}{l}0.00 \\
\pm \\
0.00 \mathrm{a}\end{array}$ & $\begin{array}{l}0.00 \pm \\
0.00 \mathrm{a}\end{array}$ & $\begin{array}{l}0.00 \pm \\
0.00 \mathrm{a}\end{array}$ \\
\hline Benzoic acid & Benzoic acid & $\mathrm{C} 7 \mathrm{H} 602$ & $\begin{array}{l}0.43 \pm \\
0.02 \mathrm{~b}\end{array}$ & $\begin{array}{l}0.00 \\
\pm \\
0.00 \mathrm{a}\end{array}$ & $\begin{array}{l}0.00 \\
\pm \\
0.00 \mathrm{a}\end{array}$ & $\begin{array}{l}0.00 \pm \\
0.00 \mathrm{a}\end{array}$ & $\begin{array}{l}0.00 \pm \\
0.00 \mathrm{a}\end{array}$ \\
\hline $\begin{array}{l}\text { Hyrocinnamic } \\
\text { acid }\end{array}$ & $\begin{array}{l}\text { Hyrocinnamic } \\
\text { acid }\end{array}$ & $\mathrm{C} 9 \mathrm{H} 10 \mathrm{O} 2$ & $\begin{array}{l}0.70 \pm \\
0.03 \mathrm{~b}\end{array}$ & $\begin{array}{l}0.00 \\
\pm \\
0.00 \mathrm{a}\end{array}$ & $\begin{array}{l}0.00 \\
\pm \\
0.00 \mathrm{a}\end{array}$ & $\begin{array}{l}0.00 \pm \\
0.00 \mathrm{a}\end{array}$ & $\begin{array}{l}0.00 \pm \\
0.00 \mathrm{a}\end{array}$ \\
\hline Cinnamic acid & Cinnamic acid & $\mathrm{C9H} 802$ & $\begin{array}{l}0.43 \pm \\
0.02 \mathrm{~b}\end{array}$ & $\begin{array}{l}0.00 \\
\pm \\
0.00 \mathrm{a}\end{array}$ & $\begin{array}{l}0.00 \\
\pm \\
0.00 \mathrm{a}\end{array}$ & $\begin{array}{l}0.00 \pm \\
0.00 \mathrm{a}\end{array}$ & $\begin{array}{l}0.00 \pm \\
0.00 a\end{array}$ \\
\hline Anethole & Anethole & $\mathrm{C} 10 \mathrm{H} 12 \mathrm{O}$ & $\begin{array}{l}2.80 \pm \\
0.15 \mathrm{c}\end{array}$ & $\begin{array}{l}0.00 \\
\pm \\
0.00 \mathrm{a}\end{array}$ & $\begin{array}{l}0.40 \\
\pm \\
0.01 \mathrm{~b}\end{array}$ & $\begin{array}{l}0.00 \pm \\
0.00 \mathrm{a}\end{array}$ & $\begin{array}{l}0.00 \pm \\
0.00 a\end{array}$ \\
\hline Octadecane & Octadecane & С18H38 & $\begin{array}{l}3.65 \pm \\
0.17 c\end{array}$ & $\begin{array}{l}0.00 \\
\pm \\
0.00 \mathrm{a}\end{array}$ & $\begin{array}{l}1.30 \\
\pm \\
0.05 \mathrm{~b}\end{array}$ & $\begin{array}{l}0.00 \pm \\
0.00 \mathrm{a}\end{array}$ & $\begin{array}{l}0.00 \pm \\
0.00 \mathrm{a}\end{array}$ \\
\hline Pentacosane & Pentacosane & $\mathrm{C} 25 \mathrm{H} 52$ & $\begin{array}{l}3.46 \pm \\
0.18 \mathrm{~b}\end{array}$ & $\begin{array}{l}0.00 \\
\pm \\
0.00 \mathrm{a}\end{array}$ & $\begin{array}{l}0.00 \\
\pm \\
0.00 \mathrm{a}\end{array}$ & $\begin{array}{l}0.00 \pm \\
0.00 \mathrm{a}\end{array}$ & $\begin{array}{l}0.00 \pm \\
0.00 \mathrm{a}\end{array}$ \\
\hline Heptadecane & Heptadecane & C27H56 & $\begin{array}{l}3.10 \pm \\
0.14 \mathrm{~b}\end{array}$ & $\begin{array}{l}3.19 \\
\pm \\
0.17 \mathrm{~b}\end{array}$ & $\begin{array}{l}0.50 \\
\pm \\
0.02 a\end{array}$ & $\begin{array}{l}0.00 \pm \\
0.00 \mathrm{a}\end{array}$ & $\begin{array}{l}0.00 \pm \\
0.00 \mathrm{a}\end{array}$ \\
\hline Queen bee acid & $\begin{array}{l}\text { 10-hydroxy-2- } \\
\text { decenoic acid }\end{array}$ & C10:1 & $\begin{array}{l}0.00 \pm \\
0.00 a\end{array}$ & $\begin{array}{l}0.00 \\
\pm \\
0.00 \mathrm{a}\end{array}$ & $\begin{array}{l}19.50 \\
\pm \\
1.70 \mathrm{~b}\end{array}$ & $\begin{array}{l}0.00 \pm \\
0.00 \mathrm{a}\end{array}$ & $\begin{array}{l}0.00 \pm \\
0.00 \mathrm{a}\end{array}$ \\
\hline Sebacic acid & decanedioic acid & C10:0 & $\begin{array}{l}0.00 \pm \\
0.00 \mathrm{a}\end{array}$ & $\begin{array}{l}0.00 \\
\pm \\
0.00 \mathrm{a}\end{array}$ & $\begin{array}{l}16.60 \\
\pm \\
1.45 \mathrm{~b}\end{array}$ & $\begin{array}{l}0.00 \pm \\
0.00 \mathrm{a}\end{array}$ & $\begin{array}{l}0.00 \pm \\
0.00 a\end{array}$ \\
\hline $\begin{array}{l}10- \\
\text { Hydroxydecanoic } \\
\text { acid }\end{array}$ & $\begin{array}{l}10- \\
\text { hydroxydecanoic } \\
\text { acid }\end{array}$ & C10:0 & $\begin{array}{l}0.00 \pm \\
0.00 \mathrm{a}\end{array}$ & $\begin{array}{l}0.00 \\
\pm \\
0.00 \mathrm{a}\end{array}$ & $\begin{array}{l}8.80 \\
\pm \\
0.73 \mathrm{~b}\end{array}$ & $\begin{array}{l}0.00 \pm \\
0.00 \mathrm{a}\end{array}$ & $\begin{array}{l}0.00 \pm \\
0.00 \mathrm{a}\end{array}$ \\
\hline $\begin{array}{l}\text { 3-hydroxy- } \\
\text { decanoic acid }\end{array}$ & $\begin{array}{l}\text { 3-hydroxy- } \\
\text { decanoic acid }\end{array}$ & C10:0 & $\begin{array}{l}0.00 \pm \\
0.00 \mathrm{a}\end{array}$ & $\begin{array}{l}0.00 \\
\pm \\
0.00 \mathrm{a}\end{array}$ & $\begin{array}{l}7.50 \\
\pm \\
0.65 \mathrm{~b}\end{array}$ & $\begin{array}{l}0.00 \pm \\
0.00 a\end{array}$ & $\begin{array}{l}0.00 \pm \\
0.00 \mathrm{a}\end{array}$ \\
\hline $\begin{array}{l}\text { 2- dodecenedioic } \\
\text { acid }\end{array}$ & $\begin{array}{l}\text { 2- dodecenedioic } \\
\text { acid }\end{array}$ & C12:1 & $\begin{array}{l}0.00 \pm \\
0.00 \mathrm{a}\end{array}$ & $\begin{array}{l}0.00 \\
\pm \\
0.00 \mathrm{a}\end{array}$ & $\begin{array}{l}19.90 \\
\pm \\
1.65 \mathrm{~b}\end{array}$ & $\begin{array}{l}0.00 \pm \\
0.00 \mathrm{a}\end{array}$ & $\begin{array}{l}0.00 \pm \\
0.00 \mathrm{a}\end{array}$ \\
\hline
\end{tabular}




\begin{tabular}{|c|c|c|c|c|c|c|c|}
\hline Common Name & Systematic Name & $\begin{array}{l}\text { Lipid } \\
\text { Numbers }\end{array}$ & $\begin{array}{l}\text { Bee } \\
\text { propolis }\end{array}$ & $\begin{array}{l}\text { Bee } \\
\text { bread }\end{array}$ & $\begin{array}{l}\text { Royal } \\
\text { jelly }\end{array}$ & Egg & Soya \\
\hline $\begin{array}{l}\text { 11-hydroxy- } \\
\text { dodecanoic acid }\end{array}$ & $\begin{array}{l}\text { 11-hydroxy- } \\
\text { dodecanoic acid }\end{array}$ & $\mathrm{C} 12: 0$ & $\begin{array}{l}0.00 \pm \\
0.00 a\end{array}$ & $\begin{array}{l}0.00 \\
\pm \\
0.00 a\end{array}$ & $\begin{array}{l}2.40 \\
\pm \\
0.13 \mathrm{~b}\end{array}$ & $\begin{array}{l}0.00 \pm \\
0.00 a\end{array}$ & $\begin{array}{l}0.00 \pm \\
0.00 a\end{array}$ \\
\hline Lauric acid & Dodecanoic acid & $\mathrm{C} 12: 0$ & $\begin{array}{l}0.00 \pm \\
0.00 a\end{array}$ & $\begin{array}{l}0.00 \\
\pm \\
0.00 \mathrm{a}\end{array}$ & $\begin{array}{l}0.00 \\
\pm \\
0.00 \mathrm{a}\end{array}$ & $\begin{array}{l}0.00 \pm \\
0.00 \mathrm{a}\end{array}$ & $\begin{array}{l}0.00 \pm \\
0.00 a\end{array}$ \\
\hline Myristic acid & $\begin{array}{l}\text { Tetradecanoic } \\
\text { acid }\end{array}$ & $\mathrm{C} 14: 0$ & $\begin{array}{l}1.89 \pm \\
0.008 \mathrm{c}\end{array}$ & $\begin{array}{l}13.43 \\
\pm 0 d\end{array}$ & $\begin{array}{l}0.00 \\
\pm \\
0.00 \mathrm{a}\end{array}$ & $\begin{array}{l}0.47 \pm \\
0.03 \mathrm{~b}\end{array}$ & $\begin{array}{l}0.30 \pm \\
0.01 \mathrm{~b}\end{array}$ \\
\hline Myristoleic acid & $\begin{array}{l}\text { 9-tetradecenoic } \\
\text { acid }\end{array}$ & C14:1n-5 & $\begin{array}{l}0.00 \pm \\
0.00 a\end{array}$ & $\begin{array}{l}0.00 \\
\pm \\
0.00 \mathrm{a}\end{array}$ & $\begin{array}{l}0.00 \\
\pm \\
0.00 \mathrm{a}\end{array}$ & $\begin{array}{l}0.15 \pm \\
0.02 b\end{array}$ & $\begin{array}{l}0.12 \pm \\
0.01 b\end{array}$ \\
\hline $\begin{array}{l}\text { Pentadecanoic } \\
\text { acid }\end{array}$ & $\begin{array}{l}\text { Pentadecanoic } \\
\text { acid }\end{array}$ & $\mathrm{C} 15: 0$ & $\begin{array}{l}0.00 \pm \\
0.00 a\end{array}$ & $\begin{array}{l}0.00 \\
\pm \\
0.00 \mathrm{a}\end{array}$ & $\begin{array}{l}0.00 \\
\pm \\
0.00 \mathrm{a}\end{array}$ & $\begin{array}{l}0.15 \pm \\
0.02 \mathrm{~b}\end{array}$ & $\begin{array}{l}0.00 \pm \\
0.00 a\end{array}$ \\
\hline Palmitic acid & $\begin{array}{l}\text { Hexadecanoic } \\
\text { acid }\end{array}$ & $\mathrm{C} 16: 0$ & $\begin{array}{l}33.50 \pm \\
2.00 \mathrm{~d}\end{array}$ & $\begin{array}{l}20.31 \\
\pm \\
1.30 \mathrm{c}\end{array}$ & $\begin{array}{l}5.00 \\
\pm \\
0.23 a\end{array}$ & $\begin{array}{l}31.37 \\
\pm \\
2.35 d\end{array}$ & $\begin{array}{l}11.36 \\
\pm \\
1.00 \mathrm{~b}\end{array}$ \\
\hline Palmitoleic acid & $\begin{array}{l}\text { 9-hexadecenoic } \\
\text { acid }\end{array}$ & C16:1n-7 & $\begin{array}{l}0.00 \pm \\
0.00 a\end{array}$ & $\begin{array}{l}0.00 \\
\pm \\
0.00 \mathrm{a}\end{array}$ & $\begin{array}{l}1.60 \\
\pm \\
0.07 c\end{array}$ & $\begin{array}{l}3.55 \pm \\
0.25 d\end{array}$ & $\begin{array}{l}0.23 \pm \\
0.02 \mathrm{~b}\end{array}$ \\
\hline $\begin{array}{l}\text { Hexadecadienoic } \\
\text { acid }\end{array}$ & $\begin{array}{l}7,10- \\
\text { hexadecadienoic } \\
\text { acid }\end{array}$ & C16:2n-6 & $\begin{array}{l}0.00 \pm \\
0.00 a\end{array}$ & $\begin{array}{l}0.00 \\
\pm \\
0.00 \mathrm{a}\end{array}$ & $\begin{array}{l}0.00 \\
\pm \\
0.00 \mathrm{a}\end{array}$ & $\begin{array}{l}0.00 \pm \\
0.00 a\end{array}$ & $\begin{array}{l}0.00 \pm \\
0.00 a\end{array}$ \\
\hline $\begin{array}{l}\text { Hexadecatrienoic } \\
\text { acid }\end{array}$ & $\begin{array}{l}7,10,13- \\
\text { hexadecatrienoic } \\
\text { acid }\end{array}$ & C16:3n-3 & $\begin{array}{l}0.00 \pm \\
0.00 a\end{array}$ & $\begin{array}{l}0.00 \\
\pm \\
0.00 \mathrm{a}\end{array}$ & $\begin{array}{l}0.00 \\
\pm \\
0.00 \mathrm{a}\end{array}$ & $\begin{array}{l}0.00 \pm \\
0.00 a\end{array}$ & $\begin{array}{l}0.00 \pm \\
0.00 \mathrm{a}\end{array}$ \\
\hline Margaric acid & $\begin{array}{l}\text { heptadecanoic } \\
\text { acid }\end{array}$ & $\mathrm{C} 17: 0$ & $\begin{array}{l}0.00 \pm \\
0.00 a\end{array}$ & $\begin{array}{l}0.00 \\
\pm \\
0.00 \mathrm{a}\end{array}$ & $\begin{array}{l}0.00 \\
\pm \\
0.00 \mathrm{a}\end{array}$ & $\begin{array}{l}0.14 \pm \\
0.03 \mathrm{~b}\end{array}$ & $\begin{array}{l}0.37 \pm \\
0.06 c\end{array}$ \\
\hline $\begin{array}{l}\text { Heptadecanoic } \\
\text { acid }\end{array}$ & $\begin{array}{l}\text { 10-heptadecenoic } \\
\text { acid }\end{array}$ & C17:1n-7 & $\begin{array}{l}0.00 \pm \\
0.00 a\end{array}$ & $\begin{array}{l}0.00 \\
\pm \\
0.00 a\end{array}$ & $\begin{array}{l}0.00 \\
\pm \\
0.00 \mathrm{a}\end{array}$ & $\begin{array}{l}0.16 \pm \\
0.02 \mathrm{~b}\end{array}$ & $\begin{array}{l}0.08 \pm \\
0.001 \mathrm{a}\end{array}$ \\
\hline Stearic acid & octadecanoic acid & C18:0 & $\begin{array}{l}0.00 \pm \\
0.00 \mathrm{a}\end{array}$ & $\begin{array}{l}0.00 \\
\pm \\
0.00 \mathrm{a}\end{array}$ & $\begin{array}{l}0.00 \\
\pm \\
0.00 \mathrm{a}\end{array}$ & $\begin{array}{l}11.00 \\
\pm \\
1.13 \mathrm{c}\end{array}$ & $\begin{array}{l}3.77 \pm \\
0.77 \mathrm{~b}\end{array}$ \\
\hline Oleic acid & $\begin{array}{l}\text { 9-Octadecenoic } \\
\text { acid }\end{array}$ & C18:1n-9 & $\begin{array}{l}27.27 \pm \\
1.450 \mathrm{~d}\end{array}$ & $\begin{array}{l}10.89 \\
\pm \\
0.94 \mathrm{~b}\end{array}$ & $\begin{array}{l}1.55 \\
\pm \\
0.03 a\end{array}$ & $\begin{array}{l}38.10 \\
\pm \\
1.75 \mathrm{e}\end{array}$ & $\begin{array}{l}20.88 \\
\pm \\
1.56 \mathrm{c}\end{array}$ \\
\hline Linoleic acid & $\begin{array}{l}9,12- \\
\text { octadecadienoic } \\
\text { acid }\end{array}$ & C18:2n-6 & $\begin{array}{l}11.04 \pm \\
0.97 \mathrm{~b}\end{array}$ & $\begin{array}{l}24.04 \\
\pm \\
1.40 \mathrm{c}\end{array}$ & $\begin{array}{l}4.45 \\
\pm \\
0.56 \mathrm{a}\end{array}$ & $\begin{array}{l}9.53 \pm \\
0.65 \mathrm{~b}\end{array}$ & $\begin{array}{l}52.70 \\
\pm \\
2.35 d\end{array}$ \\
\hline
\end{tabular}




\begin{tabular}{|c|c|c|c|c|c|c|c|}
\hline Common Name & Systematic Name & $\begin{array}{l}\text { Lipid } \\
\text { Numbers }\end{array}$ & $\begin{array}{l}\text { Bee } \\
\text { propolis }\end{array}$ & $\begin{array}{l}\text { Bee } \\
\text { bread }\end{array}$ & $\begin{array}{l}\text { Royal } \\
\text { jelly }\end{array}$ & Egg & Soya \\
\hline a-Linolenic acid & $\begin{array}{l}9,12,15- \\
\text { octadecatrienoic } \\
\text { acid }\end{array}$ & C18:3n-3 & $\begin{array}{l}8.03 \pm \\
0.54 b\end{array}$ & $\begin{array}{l}24.40 \\
\pm \\
1.30 \mathrm{c}\end{array}$ & $\begin{array}{l}8.55 \\
\pm \\
0.55 b\end{array}$ & $\begin{array}{l}0.03 \pm \\
0.001 a\end{array}$ & $\begin{array}{l}8.22 \pm \\
0.37 \mathrm{~b}\end{array}$ \\
\hline y-Linolenic acid & $\begin{array}{l}6,9,12- \\
\text { octadecatrienoic } \\
\text { acid }\end{array}$ & $C 18: 3 n-6$ & $\begin{array}{l}0.00 \pm \\
0.00 a\end{array}$ & $\begin{array}{l}0.00 \\
\pm \\
0.00 \mathrm{a}\end{array}$ & $\begin{array}{l}1.23 \\
\pm \\
0.05 b\end{array}$ & $\begin{array}{l}0.03 \pm \\
0.01 \mathrm{a}\end{array}$ & $\begin{array}{l}0.04 \pm \\
0.01 \mathrm{a}\end{array}$ \\
\hline Arachidic acid & Eicosanoic acid & C20:0 & $\begin{array}{l}0.00 \pm \\
0.00 a\end{array}$ & $\begin{array}{l}0.02 \\
\pm \\
0.01 a\end{array}$ & $\begin{array}{l}0.00 \\
\pm \\
0.00 \mathrm{a}\end{array}$ & $\begin{array}{l}0.07 \pm \\
0.02 \mathrm{a}\end{array}$ & $\begin{array}{l}0.04 \pm \\
0.02 \mathrm{a}\end{array}$ \\
\hline Paullinic acid & $\begin{array}{l}\text { 13-Eicosenoic } \\
\text { acid }\end{array}$ & C20:1n-7 & $\begin{array}{l}0.00 \pm \\
0.00 \mathrm{a}\end{array}$ & $\begin{array}{l}0.00 \\
\pm \\
0.00 \mathrm{a}\end{array}$ & $\begin{array}{l}0.00 \\
\pm \\
0.00 \mathrm{a}\end{array}$ & $\begin{array}{l}0.47 \pm \\
0.03 a\end{array}$ & $\begin{array}{l}0.00 \pm \\
0.00 \mathrm{a}\end{array}$ \\
\hline Gondoic acid & $\begin{array}{l}\text { 11-eicosenoic } \\
\text { acid }\end{array}$ & C20:1n-9 & $\begin{array}{l}0.00 \pm \\
0.00 \mathrm{a}\end{array}$ & $\begin{array}{l}0.00 \\
\pm \\
0.00 \mathrm{a}\end{array}$ & $\begin{array}{l}0.00 \\
\pm \\
0.00 \mathrm{a}\end{array}$ & $\begin{array}{l}0.03 \pm \\
0.019\end{array}$ & $\begin{array}{l}0.00 \pm \\
0.00 \mathrm{a}\end{array}$ \\
\hline $\begin{array}{l}\text { Eicosatrienoic } \\
\text { acid }\end{array}$ & $\begin{array}{l}11,14,17- \\
\text { eicosatrienoic } \\
\text { acid }\end{array}$ & $C 20: 3 n-3$ & $\begin{array}{l}0.00 \pm \\
0.00 a\end{array}$ & $\begin{array}{l}0.00 \\
\pm \\
0.00 \mathrm{a}\end{array}$ & $\begin{array}{l}0.00 \\
\pm \\
0.00 \mathrm{a}\end{array}$ & $\begin{array}{l}0.07 \pm \\
0.01 \mathrm{a}\end{array}$ & $\begin{array}{l}0.02 \pm \\
0.01 \mathrm{a}\end{array}$ \\
\hline $\begin{array}{l}\text { Dihomo-y- } \\
\text { linolenic acid }\end{array}$ & $\begin{array}{l}8,11,14- \\
\text { Eicosatrienoic } \\
\text { acid }\end{array}$ & $C 20: 3 n-6$ & $\begin{array}{l}0.00 \pm \\
0.00 a\end{array}$ & $\begin{array}{l}0.00 \\
\pm \\
0.00 \mathrm{a}\end{array}$ & $\begin{array}{l}0.00 \\
\pm \\
0.00 \mathrm{a}\end{array}$ & $\begin{array}{l}0.07 \pm \\
0.01 \mathrm{a}\end{array}$ & $\begin{array}{l}0.05 \pm \\
0.01 \mathrm{a}\end{array}$ \\
\hline Arachidonic acid & $\begin{array}{l}5,8,11,14- \\
\text { eicosatetraenoic } \\
\text { acid }\end{array}$ & $C 20: 4 n-6$ & $\begin{array}{l}0.00 \pm \\
0.00 a\end{array}$ & $\begin{array}{l}0.00 \\
\pm \\
0.00 \mathrm{a}\end{array}$ & $\begin{array}{l}0.00 \\
\pm \\
0.00 \mathrm{a}\end{array}$ & $\begin{array}{l}1.10 \pm \\
0.04 \mathrm{c}\end{array}$ & $\begin{array}{l}0.26 \pm \\
0.02 \mathrm{~b}\end{array}$ \\
\hline $\begin{array}{l}\text { Eicosapentaenoic } \\
\text { acid }\end{array}$ & $\begin{array}{l}5,8,11,14,17- \\
\text { Eicosapentaenoic } \\
\text { acid }\end{array}$ & $C 20: 5 n-3$ & $\begin{array}{l}0.00 \pm \\
0.00 \mathrm{a}\end{array}$ & $\begin{array}{l}0.00 \\
\pm \\
0.00 \mathrm{a}\end{array}$ & $\begin{array}{l}0.00 \\
\pm \\
0.00 \mathrm{a}\end{array}$ & $\begin{array}{l}0.28 \pm \\
0.02 \mathrm{~b}\end{array}$ & $\begin{array}{l}0.32 \pm \\
0.02 \mathrm{~b}\end{array}$ \\
\hline Behenic acid & Docosanoic acid & $\mathrm{C} 22: 0$ & $\begin{array}{l}3.09 \pm \\
0.17 \mathrm{~b}\end{array}$ & $\begin{array}{l}2.18 \\
\pm \\
0.11 \mathrm{~b}\end{array}$ & $\begin{array}{l}0.00 \\
\pm \\
0.00 \mathrm{a}\end{array}$ & $\begin{array}{l}0.00 \pm \\
0.00 \mathrm{a}\end{array}$ & $\begin{array}{l}0.00 \pm \\
0.00 \mathrm{a}\end{array}$ \\
\hline $\begin{array}{l}\text { Dosapentaenoic } \\
\text { acid }\end{array}$ & $\begin{array}{l}4,7,10,13,16- \\
\text { docosapentaenoic } \\
\text { acid }\end{array}$ & $C 22: 5 n-6$ & $\begin{array}{l}0.00 \pm \\
0.00 a\end{array}$ & $\begin{array}{l}0.00 \\
\pm \\
0.00 \mathrm{a}\end{array}$ & $\begin{array}{l}0.00 \\
\pm \\
0.00 \mathrm{a}\end{array}$ & $\begin{array}{l}0.27 \pm \\
0.012 b\end{array}$ & $\begin{array}{l}0.39 \pm \\
0.02 \mathrm{~b}\end{array}$ \\
\hline Cervonic acid & $\begin{array}{l}4,7,10,13,16,19- \\
\text { docosahexaenoic } \\
\text { acid }\end{array}$ & C22:6n-3 & $\begin{array}{l}0.00 \pm \\
0.00 a\end{array}$ & $\begin{array}{l}0.00 \\
\pm \\
0.00 \mathrm{a}\end{array}$ & $\begin{array}{l}0.00 \\
\pm \\
0.00 \mathrm{a}\end{array}$ & $\begin{array}{l}2.13 \pm \\
0.13 \mathrm{~b}\end{array}$ & $\begin{array}{l}0.00 \pm \\
0.00 \mathrm{a}\end{array}$ \\
\hline Total FA & Total FA & Total FA & $\begin{array}{l}99.39 \pm \\
2.80 a\end{array}$ & $\begin{array}{l}99.21 \\
\pm 3.0 \mathrm{a}\end{array}$ & $\begin{array}{l}99.28 \\
\pm \\
2.65 a\end{array}$ & $\begin{array}{l}99.20 \\
\pm 2.0 \mathrm{a}\end{array}$ & $\begin{array}{l}99.25 \\
\pm \\
2.70 a\end{array}$ \\
\hline
\end{tabular}


Principal component analysis showed that the sum of the first and second major components accounted for $73.3 \%$ total variance of the changes, with the first major component (PC1) accounting for $45.7 \%$ (eigenvalue $=16.43$ ) and the second major component $(\mathrm{PC2})$ for $27.6 \%$ (eigenvalue $=9.93$ ) (Fig. 3). The first principal component (PC1) is positively correlated with C14:1n5 (0.239), C15:0 (0.210), C16:1n7 (0.174), C17:0 (0.153), C17:1n7 (0.245), C18:0 (0.241), C18:1n9 (0.179), C20:0 (0.242), C20:1n7 (0.210), C20:1n9 (0.210), C20:3n3 (0.238), C20:3n6 (0.242), C20:4n6 (0.234), C20:5n3 (0.220), C22:5n6 (0.203), and C22:6n3 (0.210). PC1 is negatively correlated with benzoic acid $(-0.105)$, hyrocinnamic acid $(-0.105)$, cinnamic acid $(-0.105)$, anethole $(-0.123)$, octadecane $(-0.146)$, pentacosane $(0.105)$, heptadecane $(-0.165), 10$-hydroxy-2decenoic acid (-0.103), decanedioic acid (-0.103), 10-hydroxydecanoic acid (-0.103), 3-hydroxy-decanoic acid (-0.103), 2-dodecenedioic acid (-0.103), 11-hydroxy-dodecanoic acid (-0.103), a-Linolenic acid (-0.146), and docosanoic acid $(-0.146)$. The second principal component (PC2) is positively correlated with 10hydroxy-2-decenoic acid (0.269), decanedioic acid (0.269), 10-hydroxydecanoic acid (0.269), 3-hydroxydecanoic acid (0.269), 2-dodecenedioic acid (0.269), and 2-dodecenedioic acid (0.269). PC2 is negatively correlated with benzoic acid $(-0.223)$, hyrocinnamic acid $(-0.223)$, cinnamic acid $(-0.223)$, anethole $(-0.190)$, octadecane (-0.131), pentacosane (-0.223), heptadecane $(-0.201)$, palmitic acid $(-0.262)$, oleic acid $(-0.194)$, and docosanoic acid (-0.246). Royal jelly in the first quarter is closely related to 2-dodecenedioic acid, 10hydroxy-2-decenoic acid, decanedioic acid, 10-hydroxydecanoic acid, 3-hydroxy-decanoic acid, and 6,9,12octadecatrienoic acid. The soya in the second quarter is the most related to linoleic acid, oleic acid and palmitoleic acid. Bee bread and bee propolis in the 3rd quarter are extremely associated with 9,12,15octadecatrienoic acid, tetradecanoic acid, heptadecane, levulinic acid, hexadecanoic acid, octadecane, pentacosane, heptadecane, and docosanoic acid. In the 4th quarter, the whole egg is most associated with 9-octadecenoic acid, hexadecanoic acid, octadecanoic acid, 9,12-octadecadienoic acid, 9-hexadecenoic acid, 4,7,10,13,16,19-docosahexaenoic acid, and 5,8,11,14-eicosatetraenoic acid. Hydroxylated acids (like 10-hydroxy-2-decenoic, 10-hydroxydecanoic, and 3-hydroxydecanoic acids) and dicarboxylic fatty acids (like decanedioic and 2-dodecenedioic acids) are only found in royal jelly ${ }^{17}$. Myristic acid is only found in bee bread. Stearic acid finds in eggs and soya but not in bee products. Linoleic, palmitic, linolenic, and oleic acids are found in bee bread, bee propolis, royal jelly, egg, and soya but in different quantities ${ }^{18}$.

\subsection{Lipid nutritional quality}

The lipid nutritional quality of bee products including UFA, PUFA, omega-3 PUFA, SFA, omega-6 PUFA, MUFA, omega-7, omega-9, H/H, PUFA/SFA, Al, TI, NVI, and PI are stated in Table 3. Principal component analysis showed that the sum of the first and second major components accounted for $80.9 \%$ total variance of the changes, with the first major component (PC1) accounting for $50.0 \%$ (eigenvalue $=8.00$ ) and the second major component $(P C 2)$ for $30.9 \%$ (eigenvalue $=4.94$ ) (Fig. 4). The first principal component $(P C 1)$ is positively correlated with UFA (0.340), PUFA (0.325), Omega-6 (0.349), Omega-5 (0.135), Omega-6/Omega-3 (0.244), PUFA/SFA (0.352), $\mathrm{H} / \mathrm{H}(0.325), \mathrm{PI}(0.293)$, and $\mathrm{NVI}(0.241) . \mathrm{PC} 1$ is negatively correlated with SFA (-0.326), MUFA (-0.188), Omega-7 (-0.140), Al (-0.109), and TI (-0.168). The second principal component (PC2) is positively correlated with PUFA (0.138), Omega-3 (0.398), $\mathrm{Al}(0.216), \mathrm{PI}(0.127)$. PC2 is negatively correlated with MUFA (-0.292), Omega-5 (-0.392), Omega-7 (-0.350), Omega-9 (-0.334), Omega-6/Omega-3 $(-0.318), \mathrm{TI}(-0.283), \mathrm{NVI}(-0.309)$. Propolis and royal jelly in the first quarter is closely related to the Al. The bee bread in the second quarter is the most related to omega-3, PI, omega- 6 and PUFA/SFA. Egg in the 3rd 
quarter is most associated with TI, MUFA, omega-9 omega-7. In the 4th quarter, soya is most associated with omega-5, NVI, omega-6/omega-3, UFA, H/H and PUFA/SFA ${ }^{17}$. 
Table 3

Lipid nutritional quality of fatty acid from bee bread, bee propolis, and royal jelly in comparison with egg and soya.

\begin{tabular}{|c|c|c|c|c|c|}
\hline Common Name & $\begin{array}{l}\text { Bee } \\
\text { propolis }\end{array}$ & $\begin{array}{l}\text { Bee } \\
\text { bread }\end{array}$ & $\begin{array}{l}\text { Royal } \\
\text { jelly }\end{array}$ & Egg & Soya \\
\hline Saturated fatty acid (SFA) & $\begin{array}{l}38.48 \pm \\
2.25 \mathrm{~d}\end{array}$ & $\begin{array}{l}35.94 \pm \\
1.10 \mathrm{~cd}\end{array}$ & $\begin{array}{l}32.80 \pm \\
1.85 \mathrm{~b}\end{array}$ & $\begin{array}{l}43.20 \pm \\
2.50 \mathrm{e}\end{array}$ & $\begin{array}{l}15.921 \pm \\
0.90 a\end{array}$ \\
\hline Unsaturated fatty acid (UFA) & $\begin{array}{l}46.34 \pm \\
2.70 a\end{array}$ & $\begin{array}{l}59.33 \pm \\
3.40 \mathrm{~b}\end{array}$ & $\begin{array}{l}56.78 \pm \\
3.17 \mathrm{~b}\end{array}$ & $\begin{array}{l}55.99 \pm \\
3.05 b\end{array}$ & $\begin{array}{l}83.33 \pm \\
4.25 \mathrm{c}\end{array}$ \\
\hline Monounsaturated fatty acid (MUFA) & $\begin{array}{l}27.27 \pm \\
1.70 c\end{array}$ & $\begin{array}{l}10.89 \pm \\
0.75 a\end{array}$ & $\begin{array}{l}42.55 \pm \\
2.57 d\end{array}$ & $\begin{array}{l}42.47 \pm \\
2.40 \mathrm{~d}\end{array}$ & $\begin{array}{l}21.32 \pm \\
1.24 b\end{array}$ \\
\hline Polyunsaturated fatty acid (PUFA) & $\begin{array}{l}19.07 \pm \\
1.20\end{array}$ & $\begin{array}{l}48.44 \pm \\
2.70\end{array}$ & $\begin{array}{l}14.23 \pm \\
0.85\end{array}$ & $\begin{array}{l}13.52 \pm \\
0.75\end{array}$ & $\begin{array}{l}62.00 \pm \\
3.50\end{array}$ \\
\hline Omrge-3 PUFA & $\begin{array}{l}8.03 \pm \\
0.55 \mathrm{~b}\end{array}$ & $\begin{array}{l}24.40 \pm \\
1.35 \mathrm{c}\end{array}$ & $\begin{array}{l}8.55 \pm \\
0.54 \mathrm{~b}\end{array}$ & $\begin{array}{l}2.51 \pm \\
0.16 a\end{array}$ & $\begin{array}{l}8.56 \pm \\
0.53 \mathrm{~b}\end{array}$ \\
\hline Omega-6 PUFA & $\begin{array}{l}11.04 \pm \\
0.77 b\end{array}$ & $\begin{array}{l}24.04 \pm \\
1.40 c\end{array}$ & $\begin{array}{l}5.68 \pm \\
0.45 a\end{array}$ & $\begin{array}{l}11.00 \pm \\
0.70 \mathrm{~b}\end{array}$ & $\begin{array}{l}53.44 \pm \\
3.00 \mathrm{~d}\end{array}$ \\
\hline Omega-5 & $\begin{array}{l}0.00 \pm \\
0.00 \mathrm{a}\end{array}$ & $\begin{array}{l}0.00 \pm \\
0.00 a\end{array}$ & $\begin{array}{l}0.00 \pm \\
0.00 a\end{array}$ & $\begin{array}{l}0.15 \pm \\
0.01 \mathrm{~b}\end{array}$ & $\begin{array}{l}0.12 \pm \\
0.01 \mathrm{~b}\end{array}$ \\
\hline Omega-7 & $\begin{array}{l}0.00 \pm \\
0.00 \mathrm{a}\end{array}$ & $\begin{array}{l}0.00 \pm \\
0.00 \mathrm{a}\end{array}$ & $\begin{array}{l}1.60 \pm \\
0.07 \mathrm{c}\end{array}$ & $\begin{array}{l}4.19 \pm \\
0.27 d\end{array}$ & $\begin{array}{l}0.32 \pm \\
0.02 \mathrm{~b}\end{array}$ \\
\hline Omega-9 & $\begin{array}{l}27.27 \pm \\
1.75 \mathrm{~d}\end{array}$ & $\begin{array}{l}10.89 \pm \\
0.76 b\end{array}$ & $\begin{array}{l}1.55 \pm \\
0.08 a\end{array}$ & $\begin{array}{l}38.13 \pm \\
2.25 \mathrm{e}^{3}\end{array}$ & $\begin{array}{l}20.88 \pm \\
1.35 c\end{array}$ \\
\hline Omega-6/Omega-3 & $\begin{array}{l}1.37 \pm \\
0.08 \mathrm{~b}\end{array}$ & $\begin{array}{l}0.95 \pm \\
0.06 a\end{array}$ & $\begin{array}{l}0.66 \pm \\
0.05 a\end{array}$ & $\begin{array}{l}4.37 \pm \\
0.26 c\end{array}$ & $\begin{array}{l}6.24 \pm \\
0.40 \mathrm{~d}\end{array}$ \\
\hline PUFA/SFA & $\begin{array}{l}0.49 \pm \\
0.03 a\end{array}$ & $\begin{array}{l}1.35 \pm \\
0.07 \mathrm{~b}\end{array}$ & $\begin{array}{l}0.43 \pm \\
0.03 a\end{array}$ & $\begin{array}{l}0.31 \pm \\
0.02 a\end{array}$ & $\begin{array}{l}3.89 \pm \\
0.20 \mathrm{c}\end{array}$ \\
\hline Atherogenicity index (Al) & $\begin{array}{l}0.88 \pm \\
0.04 a\end{array}$ & $\begin{array}{l}1.25 \pm \\
0.05 b\end{array}$ & $\begin{array}{l}0.09 \pm \\
0.01 a\end{array}$ & $\begin{array}{l}0.59 \pm \\
0.03 a\end{array}$ & $\begin{array}{l}0.15 \pm \\
0.01 \mathrm{a}\end{array}$ \\
\hline Thrombogenicity index (TI) & $\begin{array}{l}0.80 \pm \\
0.05 b\end{array}$ & $\begin{array}{l}0.37 \pm \\
0.02 a\end{array}$ & $\begin{array}{l}0.10 \pm \\
0.01 a\end{array}$ & $\begin{array}{l}1.24 \pm \\
0.07 b\end{array}$ & $\begin{array}{l}0.24 \pm \\
0.01 \mathrm{a}\end{array}$ \\
\hline $\begin{array}{l}\text { Hypocholesterolemic/hypercholesterolemic } \\
\text { fatty acids }(H / H)\end{array}$ & $\begin{array}{l}1.30 \pm \\
0.08 a\end{array}$ & $\begin{array}{l}1.76 \pm \\
0.10 \mathrm{a}\end{array}$ & $\begin{array}{l}2.91 \pm \\
0.16 a\end{array}$ & $\begin{array}{l}1.49 \pm \\
0.08 \mathrm{a}\end{array}$ & $\begin{array}{l}7.02 \pm \\
0.35 \mathrm{~b}\end{array}$ \\
\hline Peroxidizability index (PI) & $\begin{array}{l}27.80 \pm \\
1.70 \mathrm{a}\end{array}$ & $\begin{array}{l}73.12 \pm \\
4.0 \mathrm{c}\end{array}$ & $\begin{array}{l}25.10 \pm \\
1.50 \mathrm{a}\end{array}$ & $\begin{array}{l}35.80 \pm \\
2.00 \mathrm{~b}\end{array}$ & $\begin{array}{l}75.20 \pm \\
4.40 \mathrm{c}\end{array}$ \\
\hline Nutritive value index (NVI) & $\begin{array}{l}0.81 \pm \\
0.05 \mathrm{~b}\end{array}$ & $\begin{array}{l}0.54 \pm \\
0.03 a\end{array}$ & $\begin{array}{l}0.31 \pm \\
0.02 \mathrm{a}\end{array}$ & $\begin{array}{l}1.56 \pm \\
0.09 \mathrm{~b}\end{array}$ & $\begin{array}{l}2.17 \pm \\
0.14 \mathrm{c}\end{array}$ \\
\hline
\end{tabular}

The high omega-3 diet causes a decrease in triglycerides, increases mitochondrial biogenesis, prevents inflammation, and restores insulin sensitivity. Besides, an omega-3 rich diet reduces chronic diseases such 
as obesity, diabetes, heart disease, increases blood vessels' elasticity, and prevents lipid plaques in the arteries ${ }^{20}$. Prostaglandin E3, thromboxane A3, prostacyclin 13 , and leukotriene B5 generated from the omega-3 fatty acids can suppress thrombosis and inflammation. On the other hand, prostaglandin E2, prostacyclin 12, thromboxane A2, and leukotriene B4 are produced from omega- 6 fatty acids, leading to proinflammatory and prothrombotic with the enhancement in blood viscosity ${ }^{21}$. The proportion of omega-6 / omega-3 is vital for lowering the risk of chronic diseases and preventing and managing obesity disorders. The ratio of omega-6/omega-3 lower than 3.0 is significant for healthy food. Bee products are important as sources of functional fatty acids with a well-proportioned omega- 6 to omega-3, which is a priority for dietary supplements.

The $\mathrm{H} / \mathrm{H}$ values describe the impacts of specific fatty acids on cholesterol level and metabolism. The higher proportion of $\mathrm{H} / \mathrm{H}$ directly relates to the higher content of PUFA, which is beneficial for human healthiness. The $\mathrm{P} / \mathrm{S}$ ratio is an index that expresses the lipid's nutritional quality in a specific diet. The high ratios of $\mathrm{P} / \mathrm{S}$ consider undesirable for foodstuffs due to their potentials in inducing high blood cholesterol levels. The favorable ratio of PUFA/SFA that reflects the lipid nutritional quality of a specific diet is the range of 1-2 that reduce blood cholesterol and the risk of cardiovascular diseases ${ }^{15}$.

Low Al values indicate the higher protective of foodstuffs potentials in preventing heart and coronary diseases and general and abdominal obesities and Al values. Moreover, there is a direct relationship between gestational diabetes mellitus and $\mathrm{Al}$ in pregnant women ${ }^{14}$. Myristic acid and palmitic acid consider as the most atherogenic agents. Stearic acid is a thrombogenic fatty $a \mathrm{cid}^{22}$. Foodstuffs with low TI values have a high potential to protect against coronary and heart diseases, reduce general and abdominal obesities, and prevent gestational diabetes mellitus in pregnant women. The Al and TI values of bee products are somewhat lower or similar to the Al values of egg and soya ${ }^{8}$.

The highest NVI values belong to soya, followed by egg and then bee propolis, bee bread, and royal jelly (Table 3). The NVI values of bee products range between 0.310 to 0.814 , which is lower than an egg (1.565) and soya (2.172) (Table 3). The higher NVI values in the soya and egg attribute to the higher proportion of stearic and oleic acids and the lower levels of palmitic acid ${ }^{8}$. The lowest PI value belongs to royal jelly, followed by bee propolis, egg, and bee bread, then soya oil. Royall jelly and bee propolis had a PI value similar to egg, while the PI value of bee bread was identical to soya (Table 3 ). The higher PI value of soya and bee bread can be related to higher PUFA and MUFA in the soya and bee bread oil (Table 3). The PI represents the susceptibility of fatty acid to oxidation, and this index signifies the stability of PUFA against oxidation processes. The favorable ratio of $\mathrm{PI}$ that reflects the lipid nutritional quality of a specific diet is the range of 70-90 that reduces blood cholesterol and the risk of cardiovascular diseases. All bee products in this work have PI values lower than 90. Big PI values lead to a higher level of fatty acids oxidation. However, great PI values owing to the high content of omega-3 and omega- 6 lead to more protective effects against oxidation and inflammation ${ }^{23}$.

\subsection{Inhibition of the a-amylase activity}


Bee propolis, bee bread, and royal jelly inhibit amylase with a lower level than acarbose. Kinetic parameters of amylase in the presence of acarbose and bee products measure through kinetic analysis. Figure $5 \mathrm{a}$ demonstrates plots of amylase activity at varying concentrations of acarbose. According to the LineweaverBurk plot, acarbose inhibits with a competitive inhibition trend. Since Vmax of amylase remains constant, in contrast, the $\mathrm{Km} / \mathrm{Vmax}$ and $\mathrm{Km}$ increase (Table 4). Acarbose is a competitive inhibitor of amylase activity, and as the concentration of acarbose increases, the Vmax values do not change, but the $\mathrm{Km}$ values increase (Fig. 5A). Fatty acid from bee bread (Fig. 5B), royal jelly (Fig. 5C) and, bee propolis (Fig. 5D), on the other hand, are mixed un-competitive or non-competitive inhibitors. The $\mathrm{Km} / \mathrm{Vmax}$ increases while $\mathrm{Km}$ and Vmax value decrease. By binding fatty acid to the allosteric site of the enzyme, this rises in the $\mathrm{Km} / \mathrm{Vmax}$ ratio links to the reduction in the Vmax and functional enzyme ${ }^{24}$. The active compounds in fatty acid attach to allosteric sites, avoiding starch degradation. The enzyme conformation varies when inhibitors bind to the enzyme, and the affinity of the enzyme active site for starch reduces ${ }^{25}$.

Table 4

The values of kinetic parameters ( $\mathrm{Km} / \mathrm{Vmax}, \mathrm{Km}$, Vmax) of -amylase in response to acarbose bee bread, bee propolis and royal jelly fatty acid extract.

\begin{tabular}{|llllll|}
\hline $\mathrm{a}$ & & & & & \\
Samples & Parameters & $\mathbf{0 . 0 0} \mathrm{mg} / \mathrm{ml}$ & $\mathbf{0 . 3 0} \mathrm{mg} / \mathrm{ml}$ & $\mathbf{0 . 6 0} \mathrm{mg} / \mathrm{ml}$ & $\mathbf{0 . 9 0} \mathrm{mg} / \mathrm{ml}$ \\
\hline Acarbose & $\mathrm{Km} / \mathrm{Vmax}$ & $4.78 \pm 0.25 \mathrm{a}$ & $9.07 \pm 0.52 \mathrm{~b}$ & $10.04 \pm 0.57 \mathrm{~b}$ & $13.12 \pm 0.70 \mathrm{c}$ \\
\hline Acarbose & $\mathrm{Vmax}$ & $0.73 \pm 0.04 \mathrm{a}$ & $0.74 \pm 0.05 \mathrm{a}$ & $0.70 \pm 0.04 \mathrm{a}$ & $0.68 \pm 0.03 \mathrm{a}$ \\
\hline Acarbose & $\mathrm{Km}$ & $3.47 \pm 0.20 \mathrm{a}$ & $6.74 \pm 0.35 \mathrm{~b}$ & $7.06 \pm 0.40 \mathrm{~b}$ & $8.96 \pm 0.45 \mathrm{c}$ \\
\hline Bee bread & $\mathrm{Km} / \mathrm{Vmax}$ & $5.05 \pm 0.25 \mathrm{a}$ & $7.52 \pm 0.40 \mathrm{~b}$ & $8.39 \pm 0.44 \mathrm{c}$ & $9.40 \pm 0.50 \mathrm{~d}$ \\
\hline Bee bread & $\mathrm{Vmax}$ & $0.73 \pm 0.04 \mathrm{c}$ & $0.47 \pm 0.03 \mathrm{~b}$ & $0.39 \pm 0.02 \mathrm{a}$ & $0.31 \pm 0.02 \mathrm{a}$ \\
\hline Bee bread & $\mathrm{Km}$ & $3.84 \pm 0.21 \mathrm{c}$ & $3.60 \pm 0.20 \mathrm{bc}$ & $3.29 \pm 0.18 \mathrm{ab}$ & $2.96 \pm 0.15 \mathrm{a}$ \\
\hline Bee propolis & $\mathrm{km} / \mathrm{Vmax}$ & $4.69 \pm 0.25 \mathrm{a}$ & $5.79 \pm 0.32 \mathrm{~b}$ & $6.78 \pm 0.35 \mathrm{c}$ & $7.68 \pm 0.42 \mathrm{~d}$ \\
\hline Bee propolis & $\mathrm{Vmax}$ & $0.93 \pm 0.05 \mathrm{~d}$ & $0.70 \pm 0.04 \mathrm{c}$ & $0.48 \pm 0,03 \mathrm{~b}$ & $0.36 \pm 0.02 \mathrm{a}$ \\
\hline Bee propolis & $\mathrm{Km}$ & $4.38 \pm 0.25 \mathrm{~b}$ & $4.05 \pm 0.22 \mathrm{~b}$ & $3.28 \pm 0.17 \mathrm{a}$ & $2.78 \pm 0.15 \mathrm{a}$ \\
\hline Royal jelly & $\mathrm{Km} / \mathrm{Vmax}$ & $4.21 \pm 0.23 \mathrm{a}$ & $6.71 \pm 0.35 \mathrm{~b}$ & $7.97 \pm 0.45 \mathrm{c}$ & $9.36 \pm 0.50 \mathrm{~d}$ \\
\hline Royal jelly & $\mathrm{Vmax}$ & $0.81 \pm 0.04 \mathrm{c}$ & $0.48 \pm 0,03 \mathrm{~b}$ & $0.36 \pm 0.02 \mathrm{ab}$ & $0.28 \pm 0,02 \mathrm{a}$ \\
\hline Royal jelly & $\mathrm{Km}$ & $3.43 \pm 0.20 \mathrm{c}$ & $3.23 \pm 0.17 \mathrm{bc}$ & $2.91 \pm 0.15 \mathrm{ab}$ & $2.63 \pm 0.14 \mathrm{a}$ \\
\hline
\end{tabular}

The values are expressed as means (standard deviation) of three replicates. Mean values with different letters within a row are significantly different by Tukey test at $(p<0.05)$.

\subsection{Ultraviolet and fluorescence spectroscopic analysis}

Ultraviolet-visible absorption is one of the most effective methods for monitoring enzyme conformation changes during inhibitor binding ${ }^{26}$. This study examines the absorption spectra of amylase in the presence 
of acarbose and fatty acids from bee products (Fig. 6). The ultraviolet absorption peak around $210-220 \mathrm{~nm}$ relates to the carbonyl group $\pi \pi$ transition of the peptide bond. The ultraviolet absorption peak around 255-280 $\mathrm{nm}$ attributes to the $\pi \pi$ shift of aromatic groups in the protein sequence like tryptophan, tyrosine, and phenylalanine ${ }^{16}$. Fatty acid and acarbose at these wavelengths did not have ultraviolet absorption. By increasing the concentration of acarbose and fatty acid, the ultraviolet absorption of amylase regularly increases. Accordingly, ligands such as fatty acid and acarbose can establish complexes with amylase, alter their conformation and expose the aromatic group to ultraviolet light in this manner, increasing the $\pi \pi$ transition of aromatic groups ${ }^{27}$.

Another effective technique to monitoring the interactions between amylase and inhibitors such as acarbose and fatty acid is fluorescence quenching analysis (Fig. 7). The fluorescence emission spectra of the enzyme contribute to aromatic amino acids such as tyrosine, tryptophan, and phenylalanine ${ }^{28}$. Acarbose and fatty acid did not have a fluorescence emission at this condition. The amylase exhibits a fluorescence emission peak at $360 \mathrm{~nm}$ after excitation at $280 \mathrm{~nm}$. By increasing the concentrations of acarbose and fatty acid, the fluorescence emission of amylase declines.

Furthermore, the fluorescence intensity slightly shifts toward the blue region (Fig. 7). The fluorescence quenching and shifting of amylase directly confirm the interaction between the enzyme and its inhibitors that reflect the changes in the structural architecture, conformation, surrounding environment, and polarity of the enzyme ${ }^{26}$. The molecular interactions between fatty acid or acarbose with amylase generate a nonfluorescent amylase-inhibitor complex and change the enzyme architecture and microenvironment. These effects increase the collision between fluorescent groups in the enzymes (aromatic amino acid) and quenching agents (inhibitors), leading to a decrease in the intrinsic fluorescence intensity ${ }^{28}$.

\subsection{Molecular docking simulation of amylase}

Molecular docking is a technique for predicting the binding direction of small molecules (ligands) to their protein targets, as well as their affinity. Acarbose is the competitive inhibitor of amylase, and the results of docking reveal that the interaction of acarbose with amylase occur mainly through Van der Waals interactions (with Ala106, Ala198, Arg195, Asn105, Asp197, Gly104, His101, His299, Ile235, Ile51, Leu162, Leu165, Trp58, Trp59, Tyr62, and Val107) and then hydrogen binding (with GIn63, Glu233, and Thr163 of amylase) (Table S1, Figure S1-S4 in supplemental file). The related affinity of amylase for acarbose was $8.2 \mathrm{kcal} / \mathrm{mol}$ (Table S1, Figure S1-S4 in supplemental file). Similarly, molecular docking results revealed that the interactions of fatty with amylase occurred mainly through Van der Waals interactions (Ala198, Arg10, Arg195, Arg252, Arg303, Arg398, Asn298, Asp197, Asp300, Asp356, Gln63, Gln7, Gln8, Glu233, Gly403, Gly9, His101, His201, His299, His305, Ile235, Leu162, Leu163, Leu165, Lys200, Phe335, Pro332, Pro4, Thr11, Thr163, Thr6, Trp357, Trp58, Trp59, Tyr151, Tyr62) and then hydrogen binding (with Arg421, Asp197, Asp300, Asp356, Asp402, GIn63, Glu233, Gly334, His201, Thr163, Trp59) (Table S1, Figure S1-S4 in supplemental file). But the binding pocket for fatty acid differs from the binding pocket of acarbose. The related affinity of fatty acids for acarbose ranges from -5.8 to $4.8 \mathrm{kcal} / \mathrm{mol}$, lower than the affinity of amylase for acarbose (Table S1, Figure S1-S4 in supplemental file). The highest binding affinity related to the $y$-linolenic acid and then 3-Hydroxy-decanoic acid. The fatty acid interacts with a binding pocket other 
than the active site or binding pocket near the active site. It inhibits amylase activity via an un-competitive or non-competitive inhibition strategy.

\section{Conclusions}

In summary, the differential FTIR patterns of bee bread, royal jelly, and bee propolis reflect the variations in proximate compositions and discrepancy in nutritional properties of bee bread, bee propolis, and royal jelly. The fat of bee propolis, bee bread, and royal jelly, to some extent, is identical and equal to egg fat and lower than soya. The protein of royal jelly and bee bread was higher than egg, while the propolis had lower protein than an egg. Bee propolis had the most elevated sugar, followed by bee bread and royal jelly and then soy and egg. The highest energy was found in the soya, followed by bee bread, bee propolis, royal jelly, and then egg. The higher energy of soya may be related to the higher level of lipid and protein contents. The primary fatty acid in bee propolis and bee bread were palmitic, oleic, linolenic, linoleic, behenic, and myristic acids. The primary fatty acid in royal jelly was 2-dodecenedioic, 10-hydroxy-2-decenoic, decanedioic, 10hydroxydecanoic, linolenic, 3-hydroxy-decanoic, palmitic, linoleic, 11-hydroxy-dodecanoic, oleic, palmitoleic, and $y$-linolenic acids. The bee products have a well-balanced omega- 6 to omega- 3 ratio, thrombogenicity index, atherogenicity index, PUSA/SFA, and hypocholesterolemic/hypercholesterolemic index. Besides, kinetic analyses and molecular docking reveals that fatty acids inhibit amylase activity through nucompetition or non-competition strategy. Fatty acid similar to acarbose increases ultraviolet absorption and quenches the intrinsic fluorescence intensity of amylase by forming a complex with the enzymes. In conclusion, bee products can consider as purposeful sustenance with nutritive values like egg and soya, and valuable fatty acids are essential as the non-competitive and un-competitive inhibitor for amylase intended for diabetes management.

\section{Declarations}

\section{Acknowledgments}

This study financially supports by Shiraz University (grant No. 88-GR-AGRST-108).

\section{Author contributions}

Gholamreza Kavoosi, Masoomeh Fallah, and Fatemeh Najafi conceived and designed research, conducted experiments, contributed reagents and analytical tools, and wrote the manuscript.

\section{Conflicts of interest}

The authors declare that they have no known competing for financial interests or personal relationships that could have appeared to influence the work reported in this paper.

\section{References}


1. Balica, G. et al. Potential role of propolis in the prevention and treatment of metabolic diseases. Plants, 10, 883 https://doi.org/10.3390/plants10050883 (2021).

2. Wu, F. et al. Unique dynamic mode between Artepillin $\mathrm{C}$ and human serum albumin implies the characteristics of Brazilian green propolis representative bioactive component. Sci. Rep, 10, 1-10 https://doi.org/10.1038/s41598-020-74197-4 (2020).

3. Kieliszek, M. et al. Pollen and bee bread as new health-oriented products: A review. Trends in Food Science \& Technology, 71, 170-180 https://doi.org/10.1016/j.tifs.2017.10.021 (2018).

4. Denisow, B. \& Denisow-Pietrzyk, M. Biological and therapeutic properties of bee pollen: a review. Journal of the Science of Food and Agriculture, 96, 4303-4309 https://doi.org/10.1002/jsfa.7729 (2016).

5. El-Guendouz, S., Lyoussi, B. \& Miguel, M. G. Insight into the chemical composition and biological properties of Mediterranean royal jelly. Journal of Apicultural Research, 59, 890-909 https://doi.org/10.1080/00218839.2020.1744241 (2020).

6. Yang, Y. C., Chou, W. M., Widowati, D. A., Lin, I. P. \& Peng, C. C. 10-hydroxy-2-decenoic acid of royal jelly exhibits bactericide and anti-inflammatory activity in human colon cancer cells. BMC Complementary and Alternative Medicine, 18, 1-7 https://doi.org/10.1186/s12906-018-2267-9 (2018). DOI

7. Abdelghany, A. M. et al. Profiling of seed fatty acid composition in 1025 Chinese soybean accessions from diverse ecoregions. The Crop Journal, 8, 635-644 https://doi.org/10.1016/j.cj.2019.11.002 (2020).

8. Batkowska, J., Drabik, K., Brodacki, A., Czech, A. \& Adamczuk, A. Fatty acids profile, cholesterol level, and quality of table eggs from hens fed with the addition of linseed and soybean oil. Food Chem, 334, 127612 https://doi.org/10.1016/j.foodchem.2020.127612 (2021).

9. Masuko, A. et al. Carbohydrate analysis by a phenol-sulfuric acid method in microplate format. Anal. Biochem, 339, 69-72 https://doi.org/10.1016/j.ab.2004.12.001 (2005).

10. Anantharaman, S., Padmarajaiah, N., Al-Tayar, N. G. S. \& Shrestha, A. K. Ninhydrin-sodium molybdate chromogenic analytical probe for the assay of amino acids and proteins. Spectrochimica Acta Part A: Molecular and Biomolecular Spectroscopy, 173, 897-903 https://doi.org/10.1016/j.saa.2016.10.040 (2017).

11. Mishra, S. K. et al. Rapid quantification of microalgal lipids in an aqueous medium by a simple colorimetric method. Bioresour. Technol, 155, 330-333 https://doi.org/10.1016/j.biortech.2013.12.077 (2014). DOI

12. Siahbalaei, R., Kavoosi, G. \& Noroozi, M. Protein nutritional quality, amino acid profile, anti-amylase and anti-glucosidase properties of microalgae: Inhibition and mechanisms of action through in vitro and in silico studies. LWT-Food Science and Technology, 112023 https://doi.org/10.1016/j.Iwt.2021.112023 (2021).

13. Nateghpour, B., Kavoosi, G. \& Mirakhorli, N. Amino acid profile of the peel of three citrus species and its effect on the combination of amino acids and fatty acids Chlorella vulgaris. Journal of Food Composition and Analysis, 98, 103808 https://doi.org/10.1016/j.jfca.2021.103808 (2021). 
14. Matos, A. P., Feller, R. \& Moeckel, E. H. S. Chemical characterization of six microalgae with potential utility for food application. Journal of the American Oil Chemists' Society, 93, 963-972 https://doi.org/10.1007/s11746-016-2849-y (2016). de Oliveira, J.V.Junior, A.F., Derner, R.B., \& Sant'Anna, E.S. 0

15. Canelli, G. et al. Biochemical and nutritional evaluation of Chlorella and Auxenochlorella biomasses relevant for food application. Frontiers in Nutrition, 7, 168-176 https://doi.org/10.3389/fnut.2020.565996 (2020).

16. Avwioroko, O. J. et al. Exploring the binding interactions of structurally diverse dichalcogenoimidodiphosphinate ligands with a-amylase: Spectroscopic approach coupled with molecular docking. Biochemistry and Biophysics Reports, 24, 100837 https://doi.org/10.1016/j.bbrep.2020.100837 (2020).

17. Ferioli, F., Armaforte, E. \& Caboni, M. F. Comparison of the lipid content, fatty acid profile, and sterol composition in local Italian and commercial royal jelly samples. Journal of the American Oil Chemists' Society, 91 (6), 875-884 https://doi.org/10.1007/s11746-014-2446-x (2014).

18. Kaplan, M., Karaoglu, Ã., Eroglu, N. \& Silici, S. Fatty acid and proximate composition of bee bread. Food Technology and Biotechnology, 54, 497-504 https://doi.org/10.17113/ftb.54.04.16.4635 (2016).

19. Ma, C. et al. Metabolic profiling unravels the effects of enhanced output and harvesting time on royal jelly quality. Food Res. Int, 139, 109974 https://doi.org/10.1016/j.foodres.2020.109974 (2021).

20. Simopoulos, A. P. An increase in the omega-6/omega-3 fatty acid ratio increases the risk for obesity. Nutrients, 8, 128-135 https://doi.org/10.3390/nu8030128 (2016). DOI

21. Allaj, V., Guo, C. \& Nie, D. Non-steroid anti-inflammatory drugs, prostaglandins, and cancer. Cell \& Bioscience, 3, 1-13 https://doi.org/10.1186/2045-3701-3-8 (2013).

22. Attia, Y. A., Al-Harthi, M. A., Korish, M. A. \& Shiboob, M. M. Fatty acid and cholesterol profiles and hypocholesterolemic, atherogenic, and thrombogenic indices of table eggs in the retail market. Lipid Health Disease, 14, 136-144 https://doi.org/10.1186/s12944-015-0133-z (2015).

23. Wołoszyn, J. et al. Fatty acid profiles and health lipid indices in the breast muscles of local Polish goose varieties. Poult. Sci, 99, 1216-1224 https://doi.org/10.1016/j.psj.2019.10.026 (2020).

24. Champasri, C., Phetlum, S. \& Pornchoo, C. Diverse activities and biochemical properties of amylase and proteases from six freshwater fish species. Scientific reports, 11, 1-11 https://doi.org/10.1038/s41598021-85258-7 (2021).

25. Laaroussi, H. et al. Unraveling the chemical composition, antioxidant, a-amylase, and a-glucosidase inhibition of Moroccan propolis. Food Bioscience, 101160 https://doi.org/10.1016/j.fbio.2021.101160 (2021).

26. Huang, Y., Wu, P., Ying, J., Dong, Z. \& Chen, X. D. Mechanistic study on inhibition of porcine pancreatic a-amylase using the flavonoids from dandelion. Food Chem, 344, 128610 https://doi.org/10.1016/j.foodchem.2020.128610 (2021).

27. Zheng, Y. et al. Inhibition mechanism of ferulic acid against a-amylase and a-glucosidase. Food Chem, 317, 126346 https://doi.org/10.1016/j.foodchem.2020.126346 (2020). 
28. Wang, H. et al. Interaction mechanism of carnosic acid against glycosidase (a-amylase and aglucosidase). International Journal of Biological Macromolecules, 138, 846-853 https://doi.org/10.1016/j.ijbiomac.2019.07.179 (2019). DOI

\section{Figures}

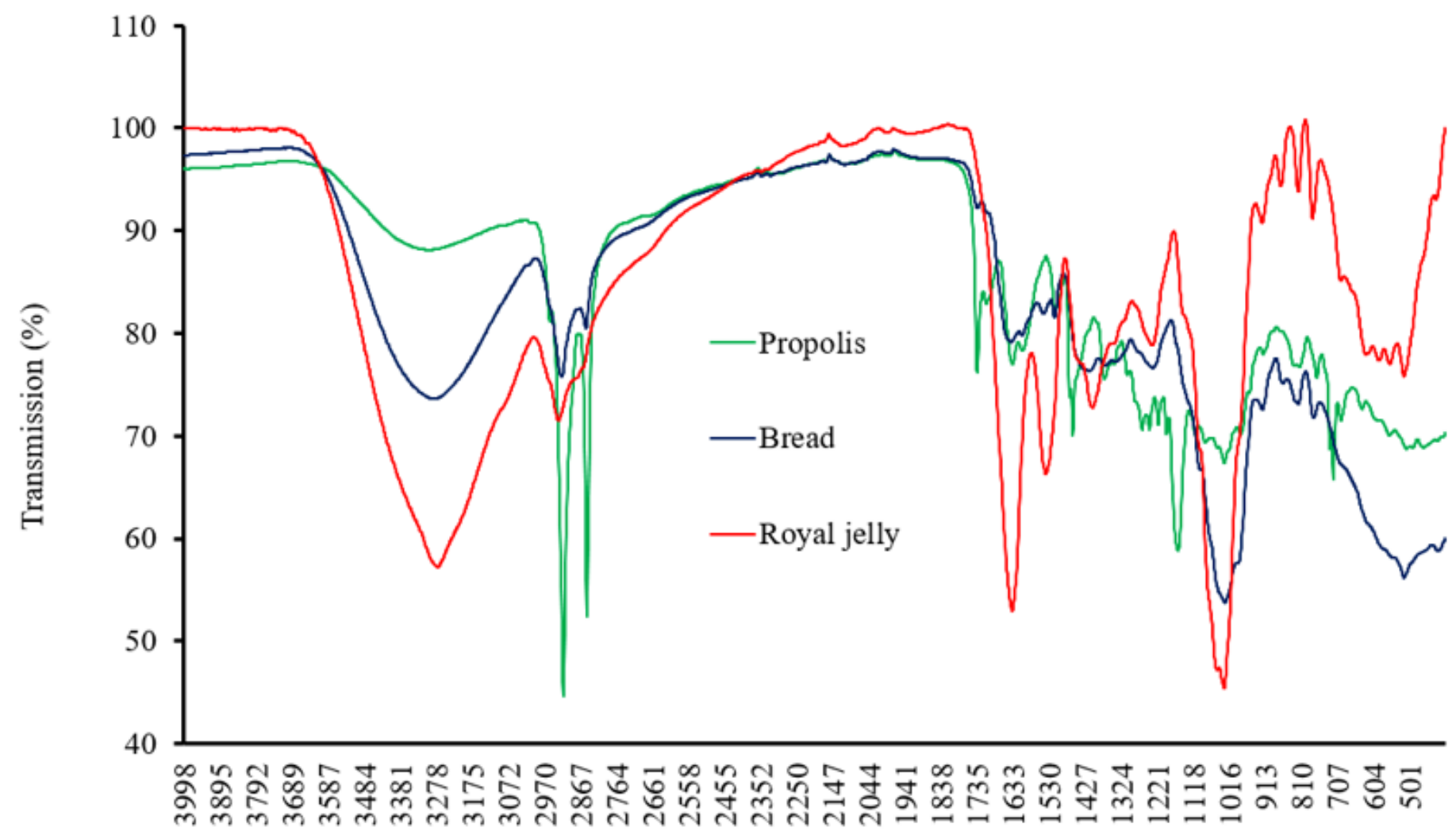

Wavenumber (cm-1)

Figure 1

Fourier transform infrared spectrum of bee bread, royal jelly and bee propolis. The differential FTIR patterns of bee bread, royal jelly and bee propolis reflect the different components in these products. 


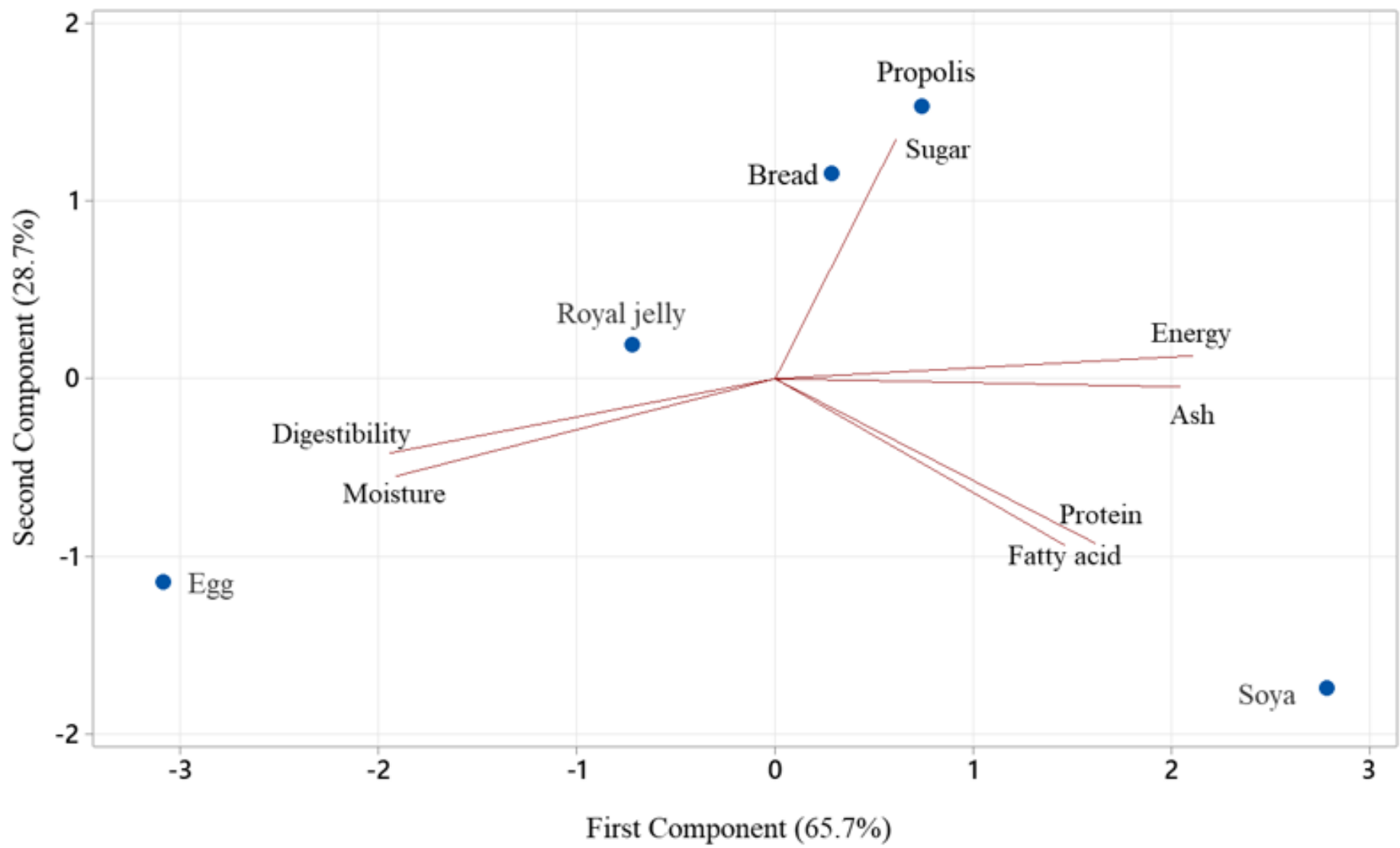

Figure 2

Principal Component Analysis (PCA) biplot illustrating the relationships among the biochemical composition of bee bread, bee propolis, royal jelly, egg and soyabean. For the abbreviation of the analyzed samples and the compounds see Table 1. 


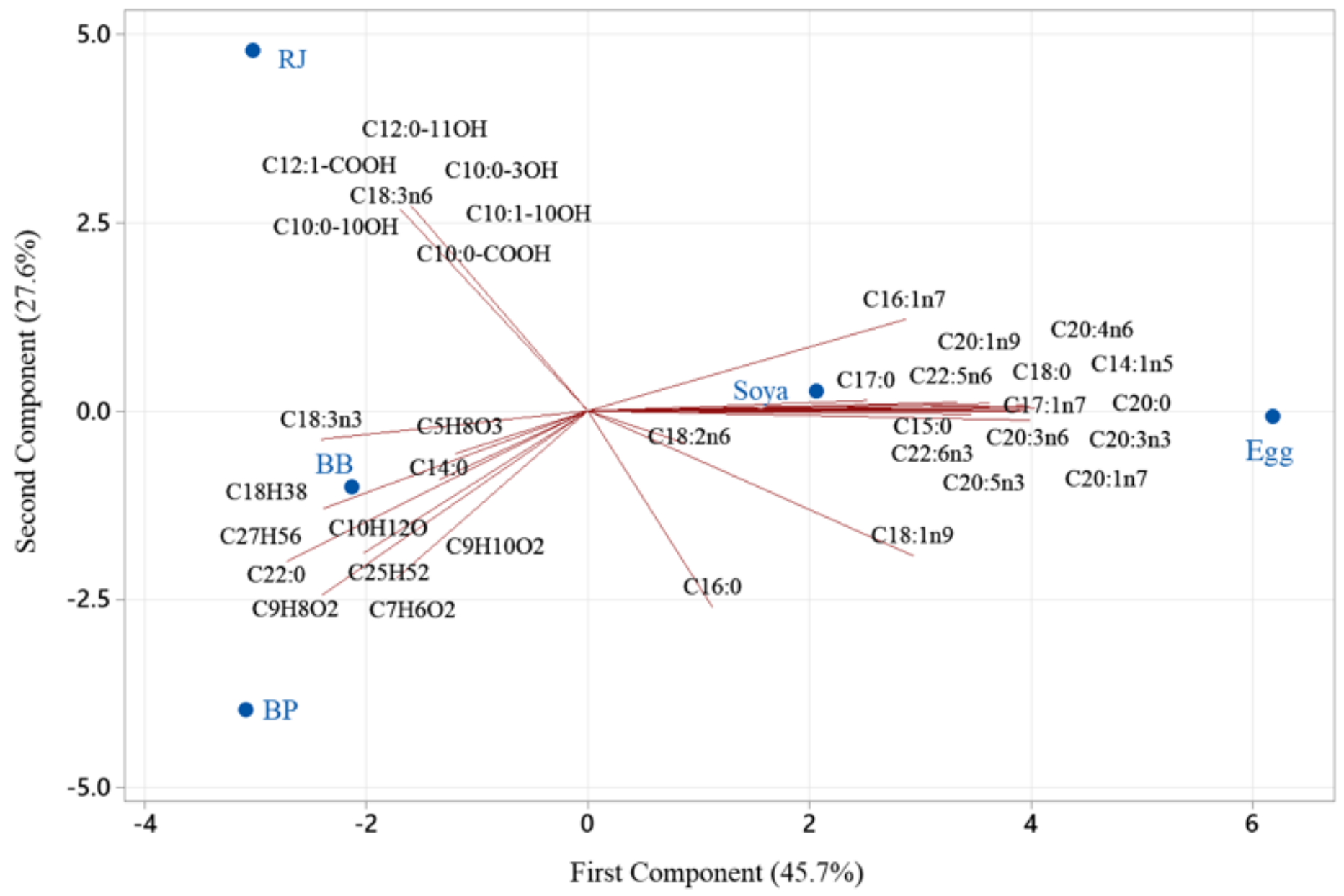

Figure 3

Principal Component Analysis (PCA) biplot illustrating the relationships among the fatty acid composition of bee bread (BB), bee propolis (BP), royal jelly (RJ), egg and soyabean. For the abbreviation of the analyzed samples and the compounds see Table 2 . 


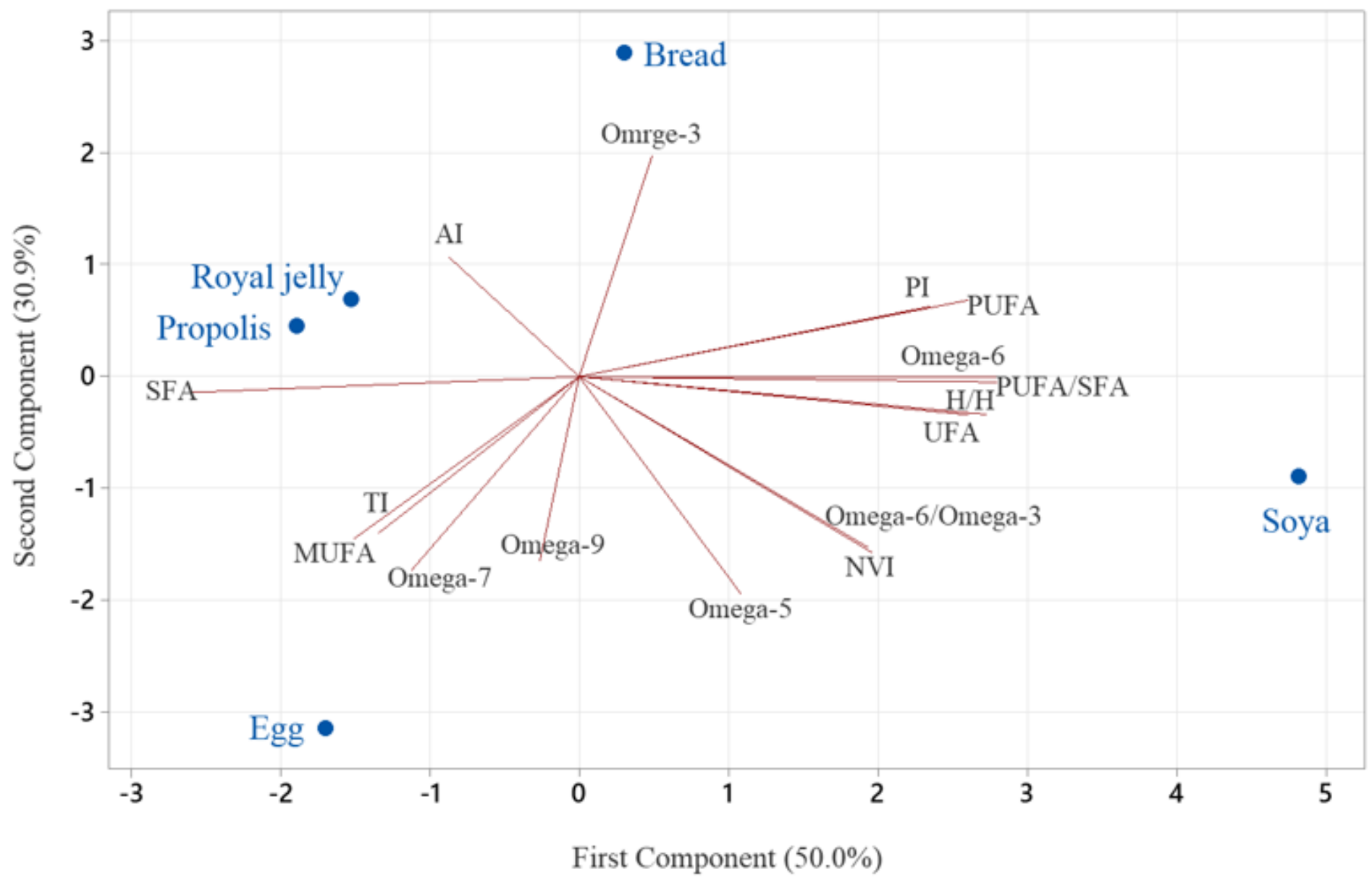

\section{Figure 4}

Principal Component Analysis (PCA) biplot illustrating the relationships among the fatty acid nutritional quality of bee bread, bee propolis, royal jelly, egg and soyabean. For the abbreviation of the analyzed samples and the compounds see Table 3 . 

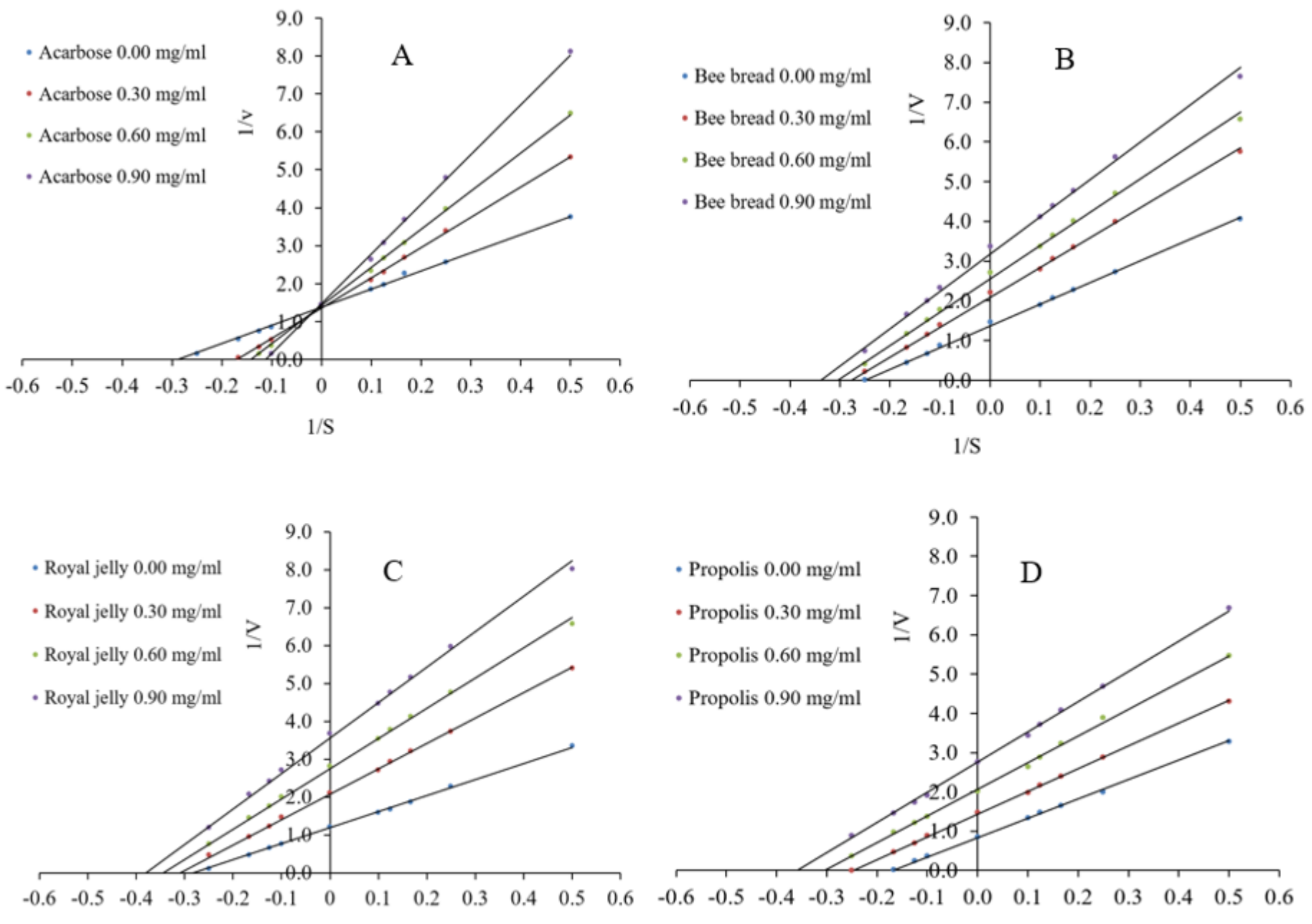

$1 / \mathrm{S}$

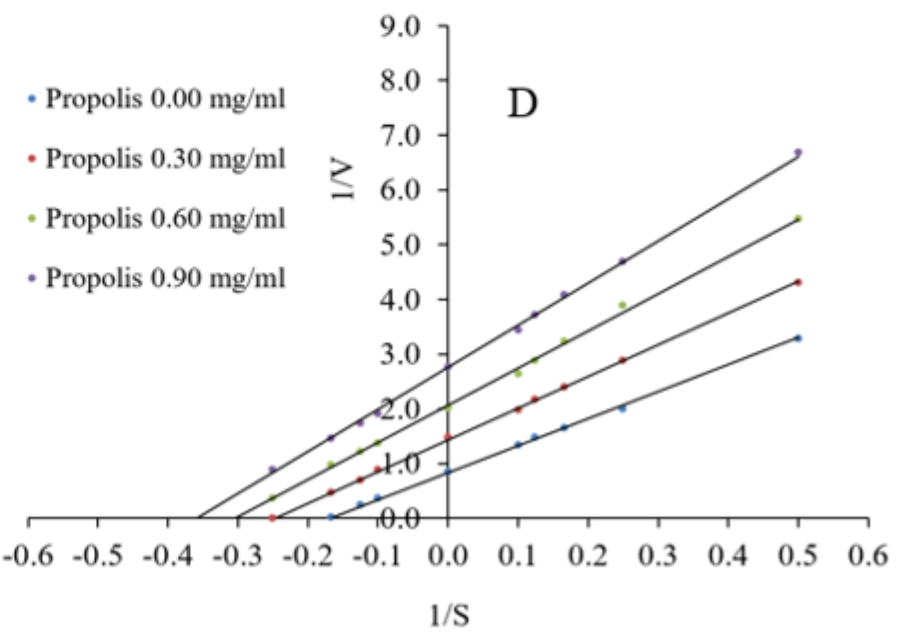

\section{Figure 5}

Double reciprocal Lineweaver-Burk plot of a-amylase activity in the absence and presence of varying concentrations of acarbose (A) and fatty acid from bee bread (B), royal jelly (C) and propolis (D). 

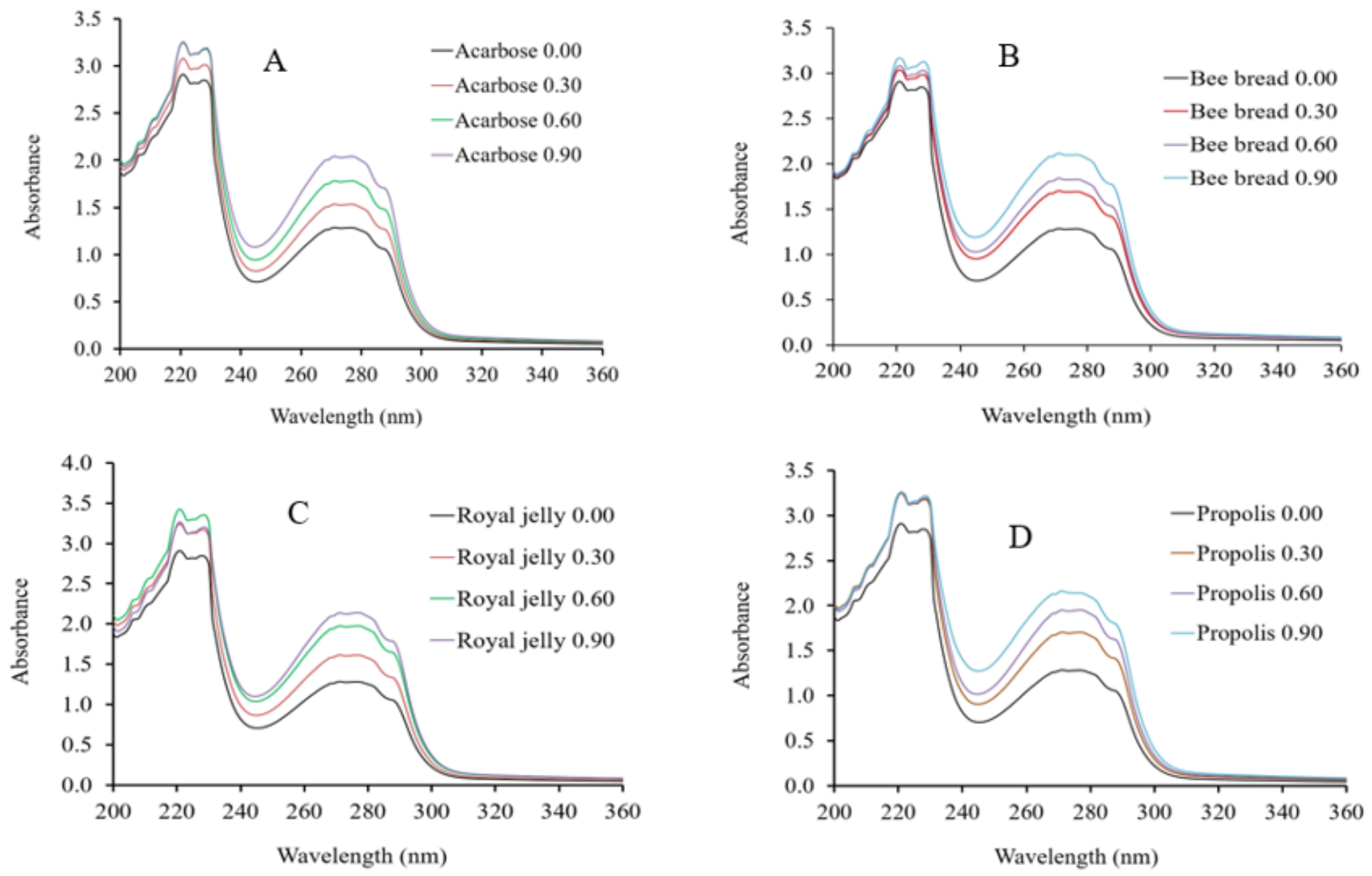

Figure 6

Ultraviolet spectra of amylase in the absence and presence of varying concentrations of acarbose (A) and fatty acid from bee bread (B), royal jelly (C) and propolis (D). 

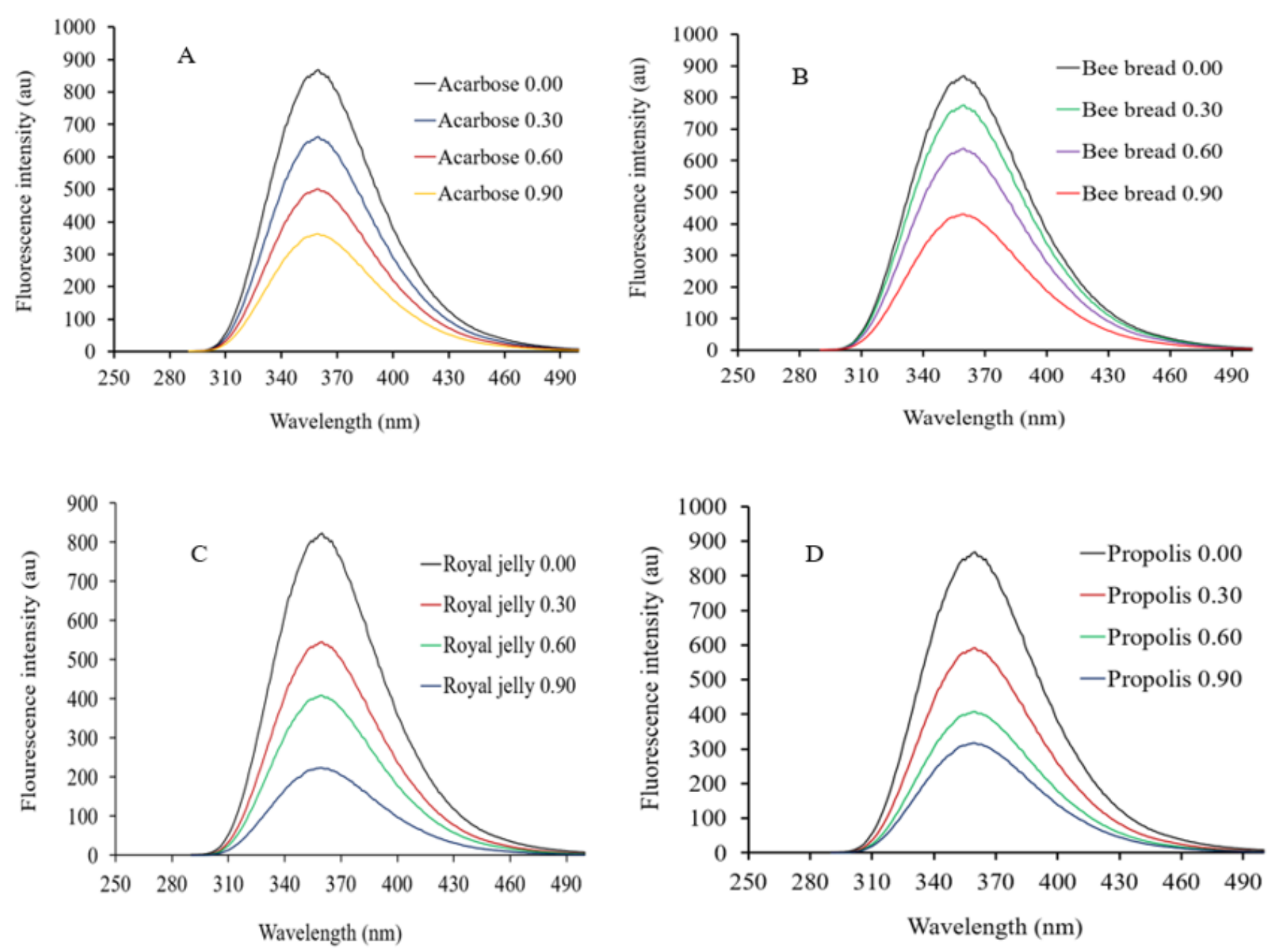

\section{Figure 7}

Fluorescence intensity of amylase in the absence and presence of varying concentrations of acarbose (A) and fatty acid from bee bread (B), royal jelly (C) and propolis (D).

\section{Supplementary Files}

This is a list of supplementary files associated with this preprint. Click to download.

- 1.propolisfattyacidmain07supplemental.docx 